COMMUNICATIONS IN

ANALYSIS AND GEOMETRY

Volume 9, Number 4, 683-723, 2001

\title{
Minimal Surfaces in a Wedge of a Slab
}

\author{
Francisco J. López ${ }^{1}$ and Francisco Martín ${ }^{1}$
}

\begin{abstract}
In this paper we construct a new family of minimal surfaces in a wedge of a slab, and study its geometrical properties in depth. These surfaces arise as a solution to Plateau's problem for some polygonal non compact boundaries.

By using these examples as barriers, we prove that any properly immersed minimal surface in a wedge of angle $\theta$ of a slab, where $\theta \in[0, \pi[$, satisfies the convex hull property. Moreover, we obtain some non existence results for properly immersed minimal surfaces with planar boundary.
\end{abstract}

\section{Introduction.}

A classical problem considered by Schwarz, Weierstrass and Riemann was to determine minimal surfaces bounded by straight lines. These authors obtained existence results for minimal surfaces with boundary a given polygon, where the sides of the polygon could be of finite or infinite length.

A comprehensive presentation of the Schwarz-Riemann-Weierstrass approach to the solution of Plateau's problem for polygonal boundaries can be found in Darboux's treatise [2, Vol. 1 and 3].

Very recently, López and Wei [9] have obtained an existence and uniqueness theorem for properly immersed minimal discs whose boundaries consist of two disjoint straight lines and a segment which meets the lines orthogonally.

In 1966, Jenkins and Serrin in [4] proved an existence and uniqueness theorem for minimal graphs bounded by straight lines. They obtained simple, necessary and sufficient conditions to solve the Dirichlet problem in a compact convex domain bounded by a polygon assuming values $+\infty,-\infty$ and continuous data on different straight segments in the boundary.

\footnotetext{
${ }^{1}$ Research partially supported by DGICYT grant number PB94-0796.
} 


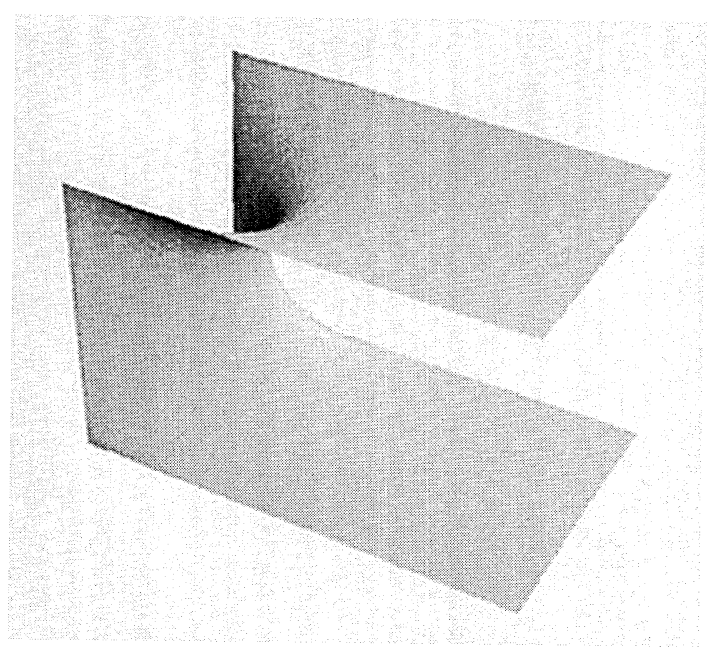

Figure 1: A Jenkins-Serrin graph.

In Figure 1, we illustrate a particular Jenkins-Serrin graph. In this case, the polygon is a rectangle and the data on the four edges are $+\infty, 0,+\infty$ and 0 , respectively.

In this paper, we construct a deformation of these particular JenkinsSerrin graphs by properly embedded minimal discs bounded by straight lines and contained in a wedge of a slab. Essentially, the deformation modifies the angle formed by the two planes containing the connected components of the boundary. In [7], the authors have obtained a natural uniqueness theorem for the surfaces in this new family. Furthermore, we use these new surfaces as barriers for maximum principle application. So, we have extended the family of minimal surfaces satisfying the convex hull property, and we have also proved some non existence results for minimal surfaces with planar boundaries.

First, we deal with the existence of properly embedded minimal surfaces whose boundary $\Gamma_{\theta d}$ consists of the following configuration of straight lines:

Fix $\theta \in[0, \pi]$ and $d \geq 0$, and consider two half-lines $r_{1}^{+}$and $r_{1}^{-}$in $\mathbb{R}^{3}$, meeting at an angle of $\theta$. If $\theta=0$ this means that the straight lines are parallel and distinct. Let $q_{1}^{+}$and $q_{1}^{-}$be two points in $r_{1}^{+}$and $r_{1}^{-}$, respectively, such that they are symmetric with respect the inner bisector of this halflines. If and only if $\theta \neq 0$, we allow $q_{1}^{+}=q_{1}^{-}$. We choose $q_{1}^{+}$and $q_{1}^{-}$in such a way that either $q_{1}^{+}=q_{1}^{-}$or the half-lines $\ell_{1}^{+}$and $\ell_{1}^{-}$on $r_{1}^{+}$and $r_{1}^{-}$starting at $q_{1}^{+}$and $q_{1}^{-}$, respectively, do not intersect. Write $d=\operatorname{dist}\left(q_{1}^{+}, q_{1}^{-}\right)$.

Let $\pi_{1}$ be the plane determined by $\ell_{1}^{+}$and $\ell_{1}^{-}$and let $\pi_{2}$ denote a plane 
parallel and distinct to $\pi_{1}$. Let $\ell_{2}^{+}$and $\ell_{2}^{-}$be the orthogonal projections to $\pi_{2}$ of $\ell_{1}^{+}$and $\ell_{1}^{-}$, respectively. Denote $q_{2}^{+}$(resp. $q_{2}^{-}$) as the orthogonal projection to $\pi_{2}$ of $q_{1}^{+}$(resp. $q_{1}^{-}$), and label $\ell_{0}^{+}$(resp. $\ell_{0}^{-}$) as the segment $\left[q_{1}^{+}, q_{2}^{+}\right]$(resp. $\left[q_{1}^{-}, q_{2}^{-}\right]$). Finally, we write

$$
\Gamma_{\theta d}^{+}=\bigcup_{i=0}^{2}\left(\ell_{i}^{+}\right), \quad \Gamma_{\theta d}^{-}=\bigcup_{i=0}^{2}\left(\ell_{i}^{-}\right)
$$

and define

$$
\Gamma_{\theta d}=\Gamma_{\theta d}^{+} \cup \Gamma_{\theta d}^{-} .
$$

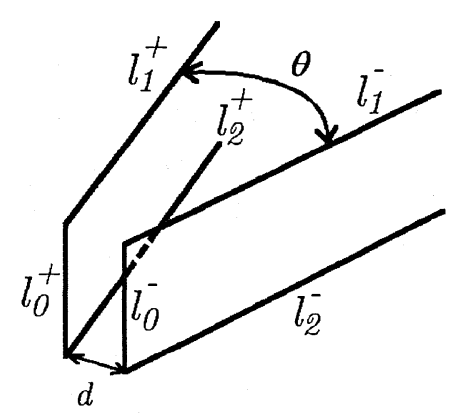

Figure 2: The curve $\Gamma_{\theta d}$.

Then, we study the following generalized Plateau's problem:

\section{Problem.}

Determine a properly immersed minimal surface $X: M \rightarrow \mathbb{R}^{3}$ satisfying:

(1) $M$ is homeomorphic to the closed unit disc $\overline{\mathbb{D}}$ minus two boundary points $E_{1}$ and $E_{2}$, that we call the ends of $M$.

(2) $X(\partial(M))=\Gamma_{\theta d}$.

(3) If $d>0, X$ is an embedding.

(4) In the limit case $\ell_{0}^{+}=\ell_{0}^{-}$(i.e., $d=0$ ), the maps $\left.X\right|_{M-\gamma^{+}}$ and $\left.X\right|_{M-\gamma^{-}}$are injective, where $\gamma^{+}$and $\gamma^{-}$are the two connected components of $\partial(M)$.

(5) $X(M)$ lies in the convex hull, $\mathcal{E}\left(\Gamma_{\theta d}\right)$, of $\Gamma_{\theta d}, \theta \in[0, \pi[$. 
(6) If $\theta=\pi, X(M)$ lies in one of the two half slab determined by $\pi_{1}, \pi_{2}$ and the plane containing $\ell_{i}^{+}, \ell_{i}^{-}, i=1,2$.

In the limit case $\theta=0$, it is known that $0<d<\|\vec{v}\|$, where $\vec{v}$ is the vector with origin $q_{1}^{+}$and end $q_{2}^{+}$, and the example is uniquely determined as one of the above mentioned Jenkins-Serrin graphs. See Remark 2, and Karcher's work [5] for a good survey.

Concerning to the general case $\theta>0$, we have proved the following:

Theorem A. There exists $d_{\theta}>0$ such that, for any $d \in\left[0, d_{\theta}\right]$, the above Plateau's problem can be solved.

The family of surfaces $\mathcal{M}$ arising from the Theorem $\mathrm{A}$ is analytical and depends on two parameters: the angle $\theta \in] 0, \pi[$ and another one $r$ which concerns to the complex structure induced by the immersion on the disc with piecewise analytical boundary. So, if we fix $\theta$, there exist $\left.r_{\theta} \in\right]-1,1[$ and a subfamily $\mathcal{M}_{\theta} \subset \mathcal{M}$ of proper, conformal, minimal immersions

$$
\left.\left.\mathcal{M}_{\theta}=\left\{X_{\theta r}: M_{\theta r} \rightarrow \mathbb{R}^{3} / r \in\right]-1, r_{\theta}\right]\right\} \subset \mathcal{M}
$$

such that $X_{\theta r}\left(\partial\left(M_{\theta r}\right)\right)=\Gamma_{\theta d(r)}$ and $\mathcal{M}=\bigcup_{\theta \in] 0, \pi[} \mathcal{M}_{\theta}$.

The opening function $\left.d:]-1, r_{\theta}\right] \rightarrow[0,+\infty[$ has the following properties:

- $d$ is analytical;

- $d$ is positive in $]-1, r_{\theta}[$;

- $d\left(r_{\theta}\right)=\lim _{r \rightarrow-1} d(r)=0$. In particular $d$ is bounded;

- $d$ has only one critical point $\left.r_{\theta}^{\prime} \in\right]-1, r_{\theta}[$, which is a maximum. Moreover, $d\left(r_{\theta}^{\prime}\right)=d_{\theta}$.

In particular, if $d \in] 0, d_{\theta}$ [, the Plateau's problem has two solutions in $\mathcal{M}_{\theta}$. If $d=0$ or $d=d_{\theta}$, the problem has a unique solution in $\mathcal{M}_{\theta}$. Finally, if $d>d_{\theta}$, there are no solutions in $\mathcal{M}_{\theta}$.

Next, we are going to make some remarks about the limits of the family of surfaces $\mathcal{M}$.

As we have mentioned above, the case $\theta=0$ leads to Jenkins-Serrin graphs. See Remark 2, and [5]. When $\theta=\pi$, the resulting family of surfaces $\left.\left.\mathcal{M}_{\pi}=\left\{X_{\pi r}: M_{\pi r} \rightarrow \mathbb{R}^{3} / r \in\right]-1, r_{\pi}\right]\right\}$ plays a special role in the Lorentz-Minkowski three dimensional space (see [6] for details). The surface 
$X_{\pi r_{\pi}}\left(M_{\pi r_{\pi}}\right)$ appeared first in [8] and motivated the discovery of the family of minimal twisted discs studied in [9].

In case $r=r_{\theta}$, the complete orientable minimal surface without boundary $\widetilde{X}_{\theta r_{\theta}}: \widetilde{M}_{\theta r_{\theta}} \rightarrow \mathbb{R}^{3}$ obtained from $X_{\theta r_{\theta}}\left(M_{\theta r_{\theta}}\right)$ by successive Schwarz reflections about straight lines is singly periodic. If in addition $\frac{\theta}{\pi} \in \mathbb{Q}$, then the induced immersion $Y_{\theta}: \widetilde{M}_{\theta} r_{\theta} /\langle T\rangle \rightarrow \mathbb{R}^{3} /\langle T\rangle$ has four ends and finite total curvature. Here $T$ is the translation by vector $2 \vec{v}$, where as above $\vec{v}$ joins the points $q_{1}^{+}$and $q_{2}^{+}$.

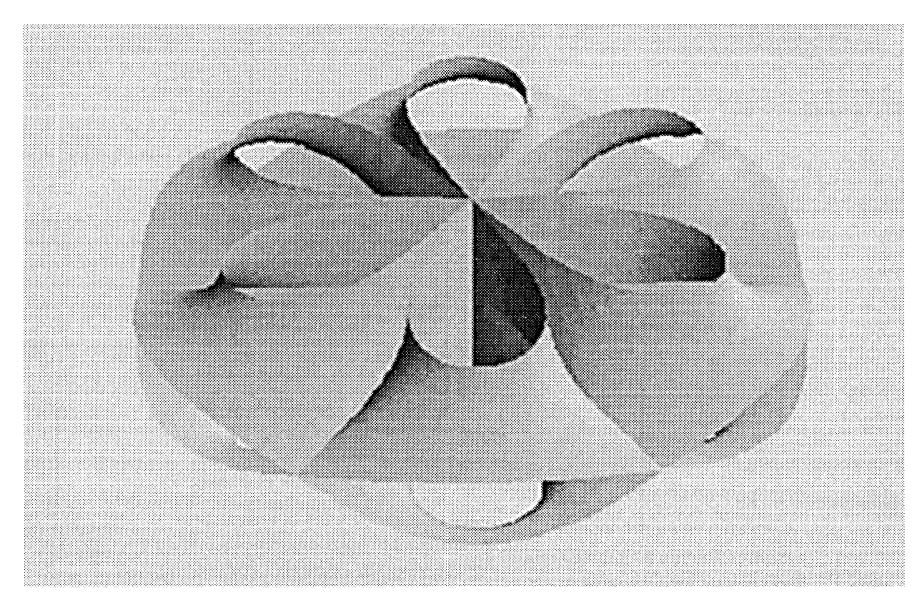

Figure 3: A fundamental piece of the surface $\tilde{X}_{\theta r_{\theta}}\left(M_{\theta r_{\theta}}\right)$ in case $\theta=\pi / 3$.

Finally, as $r \rightarrow-1$, the surfaces $X_{\theta r}\left(M_{\theta r}\right)$ converge to two parallel convex planar sectors in planes $\pi_{j}, j=1,2$, connected by the segment that joins the two vertices of the sectors. See Proposition 3.7 for details.

Hence, if we fix $\theta \in] 0, \pi]$, the one parameter family of surfaces $X_{\theta r}\left(M_{\theta r}\right)$, $\left.r \in]-1, r_{\theta}\right]$, starts at a degenerate and stable surface determined by two parallel planar sectors, and ends in the unstable example $X_{\theta r_{\theta}}\left(M_{\theta r_{\theta}}\right)$. Moreover, the spherical image of the Gauss map of the immersion $X_{\theta r}$ grows as a function of $r$. Then the geometrical and physical intuitions suggest that the immersions $\left.X_{\theta r}, r \in\right]-1, r_{\theta}^{\prime}$ ], are stable, and the immersions $X_{\theta r}$, $\left.r \in] r_{\theta}^{\prime}, r_{\theta}\right]$ are unstable. Hence, the example $X_{\theta} r_{\theta}^{\prime}$ would correspond to an almost-stable surface. 


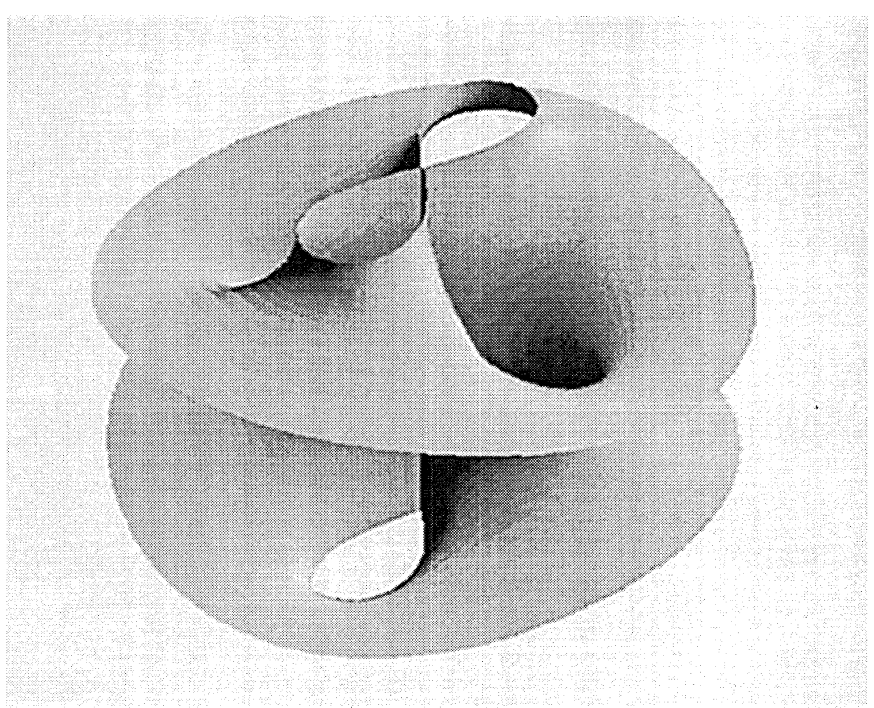

Figure 4: A fundamental piece of the surface $\widetilde{X}_{\theta r_{\theta}}\left(M_{\theta r_{\theta}}\right)$ in case $\theta=\pi / 2$.

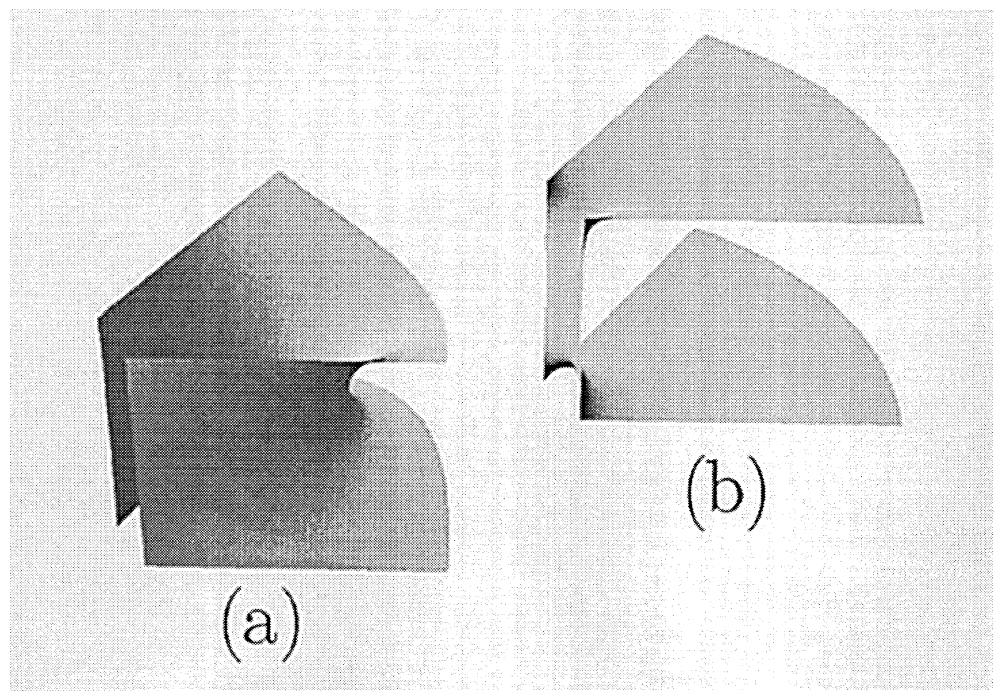

Figure 5: The two solutions in case $\theta=\pi / 3, d=0.28\|\vec{v}\|$. Figure (a) corresponds to the unstable example, and Figure (b) corresponds to the stable one. 


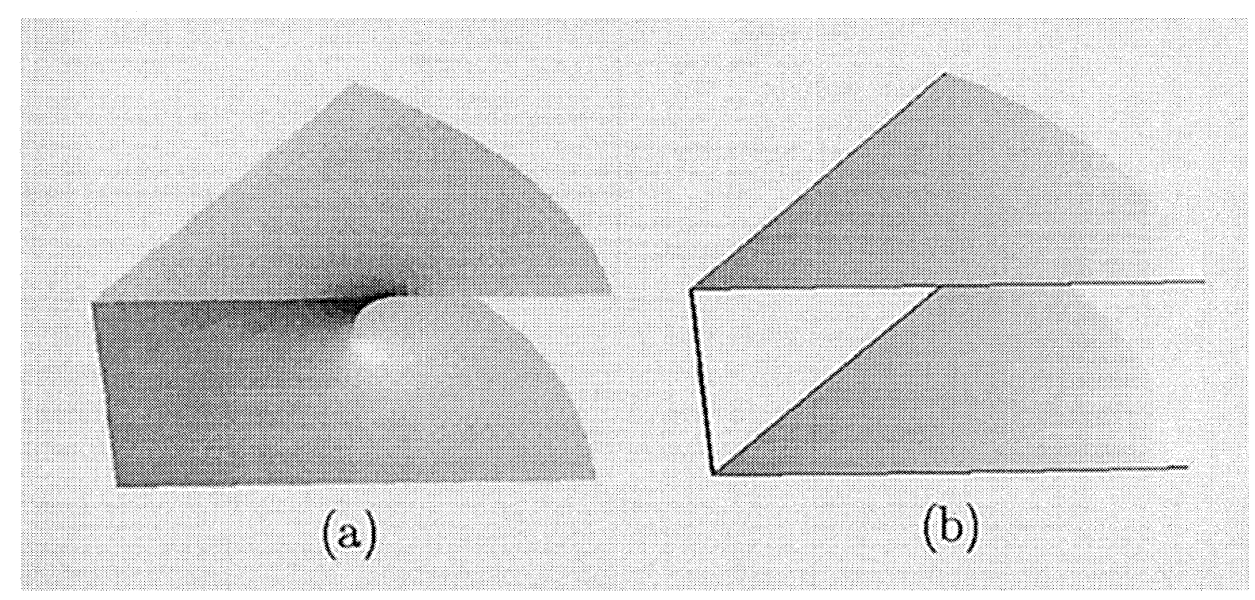

Figure 6: (a) The unstable surface $X_{\theta r_{\theta}}\left(M_{\theta r_{\theta}}\right)$, for $\theta=\pi / 3$. (b) The degenerate and stable surface determined by two parallel planar sectors, for $\theta=\pi / 3$ and $r \rightarrow-1$.

The second part of the paper is devoted to using the new examples as barriers for the maximum principle application. This kind of technique was used by Schoen [15], Hoffman-Meeks [3] and Meeks-Rosenberg [11], among others.

It is well-known that any compact minimal surface in $\mathbb{R}^{3}$ verifies the convex hull property (see for instance [13]). Recall that a surface satisfies the convex hull property if and only if it lies in the convex hull of its boundary. In this paper we have proved that this property is also satisfied by any properly immersed minimal surface (compact or not) included in a wedge of a slab.

Label $W_{\theta}$ as the solid region of $\mathbb{R}^{3}$ determined by the intersection of:

- the convex region determined by two halfplanes $P_{1}$ and $P_{2}$, meeting at an angle $\theta$ along a straight line $R$, and

- a slab $W$, which is orthogonal to $R$.

Write $F_{i}=W \cap P_{i}, i=1,2$, and $L=W \cap R$. Moreover, denote $W_{0}$ as the intersection of two orthogonal slabs. To be more precise, we have proved: 
Theorem B. Let $M$ be a properly immersed minimal surface, $M \subset W_{\theta}$, where $\theta \in[0, \pi[$. Then, $M$ is contained in the convex hull of its boundary.

Observe that we are not assuming in Theorem B that $\partial(M) \subset \partial\left(W_{\theta}\right)$. The hypothesis $M \subset W_{\theta}$ can not be substituted for the weaker one $\partial(M) \subset$ $W_{\theta}$. In Figure 7 we illustrate two examples whose boundary lies in a wedge of a slab, but none of them is contained in the convex-hull of its boundary.

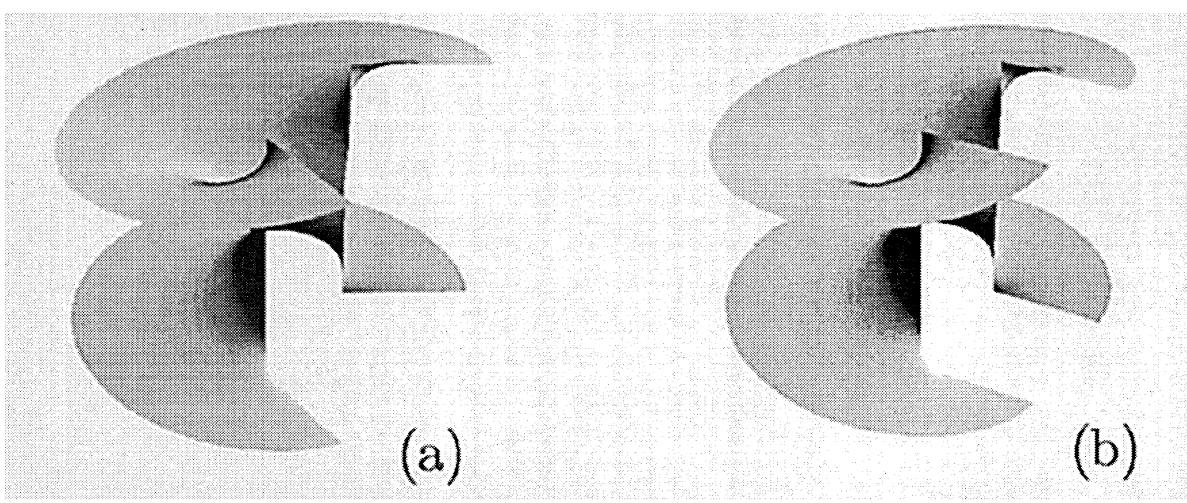

Figure 7: (a) A minimal disc bounded by $\Gamma_{\theta d}$, for $\theta=\pi / 3$ and $d=0.56\|\vec{v}\|$. (b) A minimal disc bounded by $\Gamma_{\theta d}$, for $\theta=0$ and $d=0.40\|\vec{v}\|$.

Theorem $\mathrm{B}$ does not hold for the wedges $W_{\theta}, \theta \geq \pi$, as a suitable piece of the helicoid or the surfaces in $\mathcal{M}_{\pi}$ shows (see Figure 8). In this sense it is sharp. However, it holds for surfaces $M$ lying in a wedge $\left.W_{\theta_{1}}, \theta_{1} \in\right] 0,2 \pi[$, whose boundary satisfies $\left.\partial(M) \subset W_{\theta} \subset W_{\theta_{1}}, \theta \in\right] 0, \pi[$ (see Corollary 4).

Finally, we have derived some non existence theorems for properly immersed minimal surfaces with planar boundary. Next theorem gives geometrical meaning to the number $d_{\theta}$. Let $h$ denote the thickness of the slab $W$ containing the wedge $W_{\theta}$.

Theorem C. Let $M$ be a properly immersed non flat minimal surface in a slab wedge $\left.\left.W_{\theta}, \theta \in\right] 0, \pi\right]$. Suppose that $\partial(M) \subset F_{1} \cup F_{2}$. Then,

$$
\operatorname{dist}(L, \partial(M)) \leq \frac{d_{\theta}}{2 \sin \left(\frac{\theta}{2}\right)} h .
$$




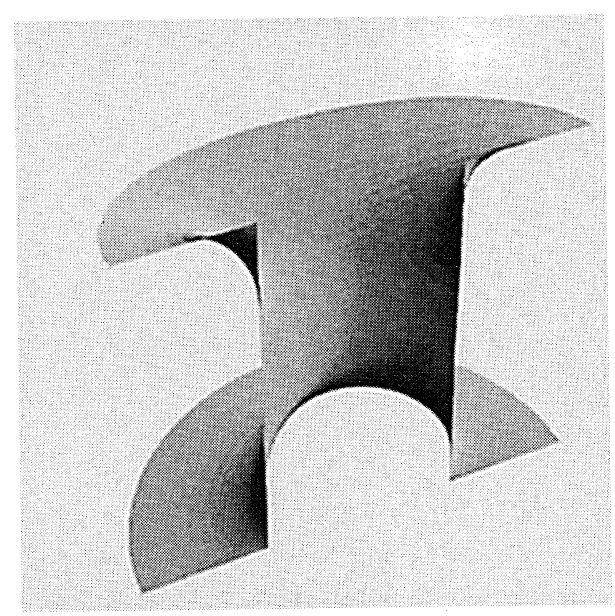

Figure 8: A surface $X_{\pi r}\left(M_{\pi r}\right)$.

The surface $X_{\theta r_{\theta}^{\prime}}\left(M_{\theta r_{\theta}^{\prime}}\right)$ corresponding to the maximum $d_{\theta}$ of the openingfunction is critical for Theorem $\mathrm{C}$, and so the inequality is sharp.

Write $\left\{C_{1}, C_{2}\right\}$ and $\left\{D_{1}, D_{2}\right\}$ as the two pairs of opposite faces of $W_{0}$.

Theorem D. Let $M$ be a properly immersed non flat minimal surface in $W_{0}$ such that $\partial(M) \subset C_{1} \cup C_{2}$. Assume that $\partial(M)$ lies in a halfspace orthogonal to $W_{0}$. Then

$$
\operatorname{dist}\left(C_{1}, C_{2}\right) \leq \operatorname{dist}\left(D_{1}, D_{2}\right) .
$$

Theorem $\mathrm{D}$ is a generalization of a classical result by Nitsche (see [12] and [14]).

This paper is laid out as follows. In Section 2, we give several results we need in this paper. In Section 3 we present the new family $\mathcal{M}$ of minimal surfaces and study its geometrical properties. In Section 4 we establish Theorems A, B, C and D.

Acknowledgements. We would like to thank A. Ros for helpful conversations. We would also like to thank Professor H. Rosenberg for suggesting that we use the new family of examples as barriers for maximum principle application.

\section{Background and Notation.}

The aim of this section is to fix the principal notation used in this paper, and to summarize some results about complete minimal surfaces. 
Let $X: M \rightarrow \mathbb{R}^{3}$ a proper conformal minimal immersion, where $M$ is a Riemann surface with piecewise analytic boundary homeomorphic to the closed unit disc $\mathbb{D}$ minus two boundary points $E_{1}$ and $E_{2}$, that we call the ends of $M$.

Remark 1. We say that $M$ is a Riemann surface with piecewise analytic boundary if and only if $M$ is a subset of an open Riemann surface $M^{\prime}$, the conformal structure of $M-\partial(M)$ is that induced by $M^{\prime}$ and $\partial(M)$ consists of a set of piecewise analytic curves. Meromorphic (resp. holomorphic) functions and 1-forms on $M$ are, by definition, the restriction of meromorphic (resp. holomorphic) functions and 1-forms on $M^{\prime}$.

The Weierstrass representation of $X$ is denoted by $(g, \eta)$. Recall that $g$ is a meromorphic function and $\eta$ a holomorphic 1-form on $M$. Both of them determine the minimal immersion $X$ as follows:

$$
X(P)=\operatorname{Re}\left(\int^{P}\left(\phi_{1}, \phi_{2}, \phi_{3}\right)\right)
$$

where

$$
\phi_{1}=\frac{1}{2}\left(1-g^{2}\right) \eta, \phi_{2}=\frac{i}{2}\left(1+g^{2}\right) \eta, \phi_{3}=g \eta
$$

are holomorphic 1-forms on $M$ satisfying:

$$
\sum_{j=1}^{3}\left|\phi_{j}\right|^{2} \neq 0
$$

Furthermore, $g$ is the stereographic projection of the Gauss map $N: M \rightarrow$ $\mathbb{S}^{2}$.

Minimal surfaces containing straight lines have special properties. Among them, we emphasize Schwarz's reflection principle (see, for instance, [13]).

A particular case of minimal surfaces bounded by straight lines was studied by Jenkins and Serrin [4]. They prove the following interesting theorem:

Theorem 1 (Jenkins, Serrin). Let $D$ be a bounded covex domain whose boundary contains two sets of open straight segments $A_{1}, \ldots, A_{k}$ and $B_{1}, \ldots, B_{l}$, with the property that no two segments $A_{i}$ and no two segments $B_{i}$ has a common endpoint. The remaining portion of the boundary consists of endpoints of the segments $A_{i}$ and $B_{i}$, and open arcs $C_{1}, \ldots, C_{m}$. Consider the Dirichlet problem: 
Determine a minimal graph in $D$ which assumes the value $+\infty$ on each $A_{i},-\infty$ on each $B_{i}$ and assigned continuous data on each of the open arcs $C_{i}$.

Let $\mathcal{P}$ denote a simple closed polygon whose vertices are chosen from among the ends points of the segments $A_{i}$ and $B_{j}$. Let $\alpha, \beta$ be, respectively, the total lenght of the segments $A_{i}$ and $B_{j}$ which are part of $\mathcal{P}$. Finally, let $\gamma$ denote the perimeter of $\mathcal{P}$.

Then, if the family of arcs $\left\{C_{i}\right\}$ is not empty, the Dirichlet problem stated above is solvable if and only if

$$
2 \alpha<\gamma \quad \text { and } 2 \beta<\gamma .
$$

Furthermore, the solution is unique if it exists.

We state two theorems which summarize the versions of the maximum principle which we require in the last section of this paper.

Theorem 2 (Interior maximum principle). Suppose $M_{1}, M_{2}$ are minimal surfaces in $\mathbb{R}^{3}$. Suppose $p$ is an interior point of both $M_{1}$ and $M_{2}$, and suppose $T_{p} M_{1}=T_{p} M_{2}$. Assume that $T_{p} M_{1}=\left\{x_{3}=0\right\}$ so that both $M_{1}$, $M_{2}$ are given near $p$ as the graphs of two real analytic functions $u_{1}$ and $u_{2}$, respectively. If $u_{1} \geq u_{2}$ in a neighbourhood of $p$, then $M_{1}=M_{2}$.

A elementary consequence of this result is the nonexistence of compact nonplanar minimal surfaces with boundary contained in a plane $\Pi$. Meeks and Rosenberg proved that this result remains true in the non compact case if in addition the surface lies in the slab determined by $\Pi$ and a plane $\Pi^{\prime}$ parallel to $\Pi$. In fact, they obtained the following:

Theorem 3 (Meeks, Rosenberg). Suppose $M \subset\left\{\left(x_{1}, x_{2}, x_{3}\right) \in \mathbb{R}^{3} \quad\right.$ : $\left.x_{3} \geq 0\right\}$ is a properly immersed minimal surface with nonempty, possibly noncompact, boundary $\partial(M)$. If $x_{3}(\partial(M)) \geq \delta$, then $x_{3}(M) \geq \delta$.

See [11, Lemma 2.1] for details.

\section{The existence results.}

In this section we construct the family of minimal surfaces described in the introduction. Moreover, we present in successive subsections a complete study of the main geometrical properties of these examples. 


\subsection{The Weierstrass representation and symmetry of the new family of examples.}

Take $n \in[1,2]$ and $r \in]-1,1\left[\right.$. Put $\left.r=-\cos \left(x_{0}\right), x_{0} \in\right] 0, \pi[$. Consider the Riemann surface

$$
\mathcal{N}=\left\{(z, w) \in \mathbb{C}^{*} \times \mathbb{C} / w^{2}=\left(z-e^{i x_{0} / n}\right)\left(z-e^{-i x_{0} / n}\right)\right\}
$$

and define in the $z$-plane:

$$
\begin{aligned}
& \left.\left.\left.\left.s_{1}^{+}=\left\{\lambda e^{-\frac{\pi i}{n}}: \lambda \in\right] 0,1\right]\right\}, \quad s_{1}^{-}=\left\{\lambda e^{\frac{\pi i}{n}}: \lambda \in\right] 0,1\right]\right\}, \\
& s_{2}^{+}=\left\{\lambda e^{-\frac{\pi i}{n}}: \lambda \in\left[1,+\infty[\}, \quad s_{2}^{-}=\left\{\lambda e^{\frac{\pi i}{n}}: \lambda \in[1,+\infty[\}\right.\right.\right. \\
& s_{0}^{+}=\left\{e^{-i t}: t \in\left[x_{0} / n, \pi / n\right]\right\}, \quad s_{0}^{-}=\left\{e^{i t}: t \in\left[x_{0} / n, \pi / n\right]\right\} .
\end{aligned}
$$

Then, label $C \subset \mathcal{N}$ as the connected component of $z^{-1}\left(\mathbb{C}-\left(\cup_{i=0}^{2}\left(s_{i}^{+} \cup s_{i}^{-}\right)\right)\right)$ containing the point

$$
P_{0}=\left(1,+\sqrt{2\left(1-\cos \left(x_{0} / n\right)\right)}\right)
$$

Define

$$
M=\bar{C}
$$

where $\bar{C}$ means the closure of $C$ in $\mathcal{N}$.

Finally, label $\gamma_{i}^{+}=z^{-1}\left(s_{i}^{+}\right), \gamma_{i}^{-}=z^{-1}\left(s_{i}^{-}\right), i=0,1,2$. Denote

$$
\gamma^{+}=\bigcup_{i=0}^{2} \gamma_{i}^{+}, \quad \gamma^{-}=\bigcup_{i=0}^{2} \gamma_{i}^{-}
$$

It is clear that $\partial(M)=\gamma^{+} \cup \gamma^{-}$. Furthermore, note that $\left.z\right|_{\gamma_{i}^{+}}$and $\left.z\right|_{\gamma_{i}^{-}}$ are bijective maps onto $s_{i}^{+}$and $s_{i}^{-}$, respectively, $i=1,2$. However, $\gamma_{0}^{+}$and $\gamma_{0}^{-}$consist of two copies of $s_{0}^{+}$and $s_{0}^{-}$, respectively. See Figure 9 for more details.

Since $z(M)$ is simply connected and $0 \notin z(M)$, then the function $z^{n}+$ $z^{-n}+2 r$ is well defined on $M$. We choose the branch of $z^{n}$ satisfying $1^{n}=1$.

This choice of the branch of $z^{n}$ implies that the function $z^{n}+z^{-n}+2 r$ has neither zeroes nor poles on $M-\partial(M)$. 


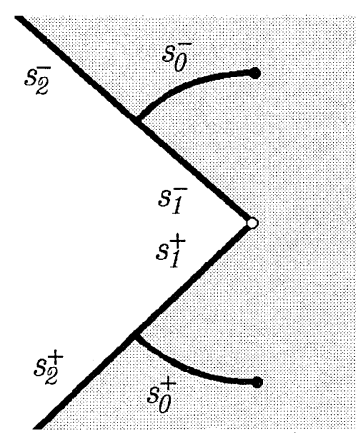

(a)

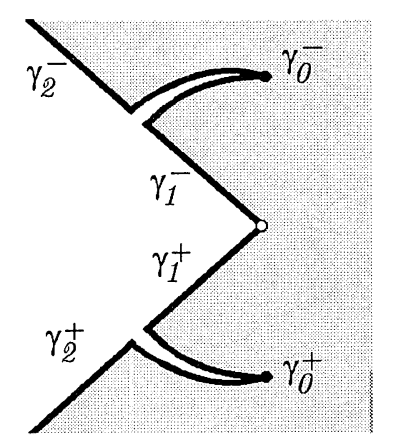

(b)

Figure 9: (a) The domain $z(M)$. (b) The surface $M$.

Hence, the function $\varphi((z, w))=\sqrt{z^{n}+z^{-n}+2 r}$ has a well defined branch on the (simply connected) domain $M-\partial(M)$, that can be extended continuously to $M$. For convenience, we choose the branch verifying $\varphi\left(P_{0}\right)<0$. Moreover, note that given $z_{0} \in\left(s_{0}^{+} \cup s_{0}^{-}\right)$and denoting $\left\{P^{+}, P^{-}\right\}=z^{-1}\left(z_{0}\right)$, we have $\varphi\left(P^{+}\right)=-\varphi\left(P^{-}\right)$.

We consider the meromorphic data on $M$

$$
g=i z, \quad \phi_{3}=B \frac{d z}{z \varphi}
$$

where $B>0$.

For simplicity, we write

$$
\tau_{r}=\frac{d z}{z \varphi}
$$

and as usual, we denote

$$
\left(\phi_{1}, \phi_{2}, \phi_{3}\right)=\frac{B}{2}\left(-i(1 / z+z) \tau_{r},(1 / z-z) \tau_{r}, \tau_{r}\right) .
$$

As $M$ is homeomorphic to a closed disc minus two boundary points, then

$$
X: M \longrightarrow \mathbb{R}^{3}
$$

$$
X(P)=\operatorname{Re} \int_{P_{0}}^{P}\left(\phi_{1}, \phi_{2}, \phi_{3}\right)
$$

is a well defined conformal minimal immersion of $M$ in $\mathbb{R}^{3}$. 
Remark 2. If $n=2$ and $r \in]-1,1[$, the immersions associated to the Weierstrass data (4) are known and correspond to some Jenkins-Serrin graphs (see Figure 1). Following the notation of [4] and Theorem 2, these minimal graphs are the only with boundary values $+\infty, 0,+\infty, 0$ on a rectangle.

Extension by Schwarz reflections of these surfaces gives embedded doubly-periodic examples with two orthogonal planes of symmetry between adjacent saddle towers. As the quotient of such a surface under its group of translations has finite topology, then this quotient has finite total curvature (see Meeks-Rosenberg work [10]).

Karcher [5] proved that the arising Weierstrass data are those described in (4).

For the reasons explained in last remark, we are going to restrict our attention to the case $n<2$, and in what follows we suppose $n \in[1,2[$.

Concerning to its symmetries, let $S_{h}, S_{v}$ denote the antiholomorphic transformations on $M$

$$
S_{h}((z, w))=(1 / \bar{z}, \bar{w} / \bar{z}), \quad S_{v}((z, w))=(\bar{z}, \bar{w}) .
$$

Notice that $S_{h}\left(P_{0}\right)=S_{v}\left(P_{0}\right)$, and

$$
\begin{aligned}
g \circ S_{h} & =1 / \bar{g}, g \circ S_{v}=-\bar{g}, \\
S_{h}^{*}\left(\phi_{3}\right) & =-\overline{\phi_{3}}, S_{v}^{*}\left(\phi_{3}\right)=\overline{\phi_{3}} ;
\end{aligned}
$$

so elementary arguments imply that $S_{h}$ (resp. $S_{v}$ ) induces on $X(M)$ a symmetry with respect to the plane $x_{3}=0$ (resp. $\left.x_{1}=0\right)$.

\subsection{The boundary behaviour.}

The following step is to study the behaviour of $X$ along $\partial(M)$.

First, observe that

$$
\begin{gathered}
S_{v}\left(\gamma_{i}^{+}\right)=\gamma_{i}^{-}, i=0,1,2 \\
S_{h}\left(\gamma_{1}^{+}\right)=\gamma_{2}^{+}, S_{h}\left(\gamma_{1}^{-}\right)=\gamma_{2}^{-}, S_{h}\left(\gamma_{0}^{+}\right)=\gamma_{0}^{+}, S_{h}\left(\gamma_{0}^{-}\right)=\gamma_{0}^{-} .
\end{gathered}
$$

Introduce the following notation. Let $\ell_{i}^{+}=X\left(\gamma_{i}^{+}\right)$and $\ell_{i}^{-}=X\left(\gamma_{i}^{-}\right), i=$ $0,1,2$, and label

$$
\Gamma=\bigcup_{i=0}^{2}\left(\ell_{i}^{+} \cup \ell_{i}^{-}\right) .
$$

We can prove the following: 
Lemma 1. The maps $\left.X\right|_{\gamma^{+}},\left.X\right|_{\gamma^{-}}$are injective, and:

1. The curves $\ell_{1}^{+}$and $\ell_{1}^{-}$are half-lines contained in a plane $x_{3}=k, k>0$. Furthermore, they are symmetric with respect to plane $x_{1}=0$, and the straight lines containing $\ell_{1}^{+}$and $\ell_{1}^{-}$meet at an angle $\theta=(2-n) \pi / n$.

2. The curve $\ell_{2}^{+}$(resp. $\ell_{2}^{-}$) is the image of $\ell_{1}^{+}$(resp. $\ell_{1}^{-}$) under the symmetry with respect to plane $x_{3}=0$.

3. The functions $\left.x_{1}\right|_{\ell_{i}^{+}}$and $\left.x_{1}\right|_{\ell_{i}^{-}}$diverge to $+\infty$ and $-\infty$, respectively, $i=1,2$.

If $n \in] 1,2\left[\right.$, then $\left.x_{2}\right|_{\ell_{i}^{+}},\left.x_{2}\right|_{\ell_{i}^{-}}$diverge to $+\infty, i=1,2$. In case $n=1$, $\left.x_{2}\right|_{\ell_{i}^{+}}$and $\left.x_{2}\right|_{\ell_{i}^{-}}$are constant.

4. The curve $\ell_{0}^{+}$(resp. $\ell_{0}^{-}$) is the vertical segment joining the end points of $\ell_{1}^{+}$and $\ell_{2}^{+}$(resp. $\ell_{1}^{-}$and $\left.\ell_{2}^{-}\right)$.

Proof. On $\gamma_{1}^{-}$, put $\left.\left.z=t e^{\pi i / n}, t \in\right] 0,1\right]$. Taking into account that $\varphi\left(P_{0}\right)<0$, an analytic continuation argument gives:

$$
\begin{aligned}
\phi_{3}(t) & =-i B \frac{d t}{\left|t \sqrt{t^{n}+t^{-n}-2 r}\right|} \\
\phi_{1}(t) & =-\frac{i}{2}((1 / t+t) \cos (\pi / n)+i(t-1 / t) \sin (\pi / n)) \phi_{3}(t) \\
\phi_{2}(t) & =\frac{1}{2}((1 / t-t) \cos (\pi / n)-i(t+1 / t) \sin (\pi / n)) \phi_{3}(t) .
\end{aligned}
$$

These equations imply that $\ell_{1}^{-}$is a half-line contained in a straight line $x_{3}=k, x_{2}-\tan (\pi / n) x_{1}=k^{\prime}$, for suitable $k, k^{\prime} \in \mathbb{R}$. Note that this straight line meets the straight line $x_{3}=k, x_{1}=0$ at an angle of $\frac{(2-n) \pi}{2 n}$. Notice also that $\operatorname{Re}\left(\phi_{1}(t) / d t\right)>0$, and so $\left.X\right|_{\gamma_{1}^{-}}$is injective.

Moreover, it is clear that $\left.x_{1}\right|_{\ell_{1}^{-}}$diverges to $-\infty$. If $n>1$, then $\left.x_{2}\right|_{\ell_{1}^{-}}$ diverges to $+\infty$, and $n=1$ implies that $\left.x_{2}\right|_{\ell_{1}^{-}}$is constant.

The curve $\gamma_{0}^{-}$consists of two copies, $\delta_{1}$ and $\delta_{2}$, of $s_{0}^{-}$. We can assume that $\delta_{1}(t)$ and $\delta_{2}(t)$ are the two lifts to $M$ of the curve $e^{i t / n}, t \in\left[x_{0}, \pi\right]$, in the $z$-plane, satisfying $\delta_{1}(\pi) \in \gamma_{1}^{-}$and $\delta_{2}(\pi) \in \gamma_{2}^{-}$, respectively.

Let $\delta(t)$ be the lift to $M$ of the curve $e^{i t / n}, t \in\left[0, x_{0}\right]$, in the $z$-plane. Observe $\delta(0)=P_{0}$ and $\delta\left(x_{0}\right)=\delta_{1}\left(x_{0}\right)=\delta_{2}\left(x_{0}\right)$.

Taking our choice of branches into account, we have

$$
\varphi(\delta(t))<0, \quad i \varphi\left(\delta_{1}(t)\right) \in \mathbb{R}^{-}, \quad i \varphi\left(\delta_{2}(t)\right) \in \mathbb{R}^{+} .
$$


Then, it is straightforward to check that:

$$
\operatorname{Re}\left(\phi_{i}\left(\frac{d \delta_{j}}{d t}\right)\right)=0, i=1,2, j=1,2,
$$

and so $\ell_{0}^{-}$is a vertical segment.

Furthermore, note that:

$$
\operatorname{Re}\left(\phi_{3}\left(\frac{d \delta}{d t}\right)\right)=0, \phi_{3}\left(\frac{d \delta_{1}}{d t}\right) \in \mathbb{R}^{+}, \phi_{3}\left(\frac{d \delta_{2}}{d t}\right) \in \mathbb{R}^{-}
$$

and this implies that $k>0$ and $\left.X\right|_{\gamma_{0}^{-}}$is injective.

Taking into account the symmetries induced by $S_{h}$ and $S_{v},(7)$ and (8), it is not hard to conclude the proof.

Remark 3. Note that the angle function $\theta:[1,2[\rightarrow] 0, \pi], \theta(n)=(2-n) \pi / n$, is an analytical diffeomorphism. Hence, we can use $(\theta, r)$ instead of $(n, r)$ to parametrize our family of surfaces.

In the following, we label the plane $x_{3}=k$ as $\pi_{1}$ and the plane $x_{3}=-k$ as $\pi_{2}$.

Moreover, and in the remainder of this section, we fix $\theta \in] 0, \pi]$ (or $n \in$ $[1,2[)$.

Consider $\delta_{1}, \delta_{2}$ and $\delta$ as in the proof of Lemma 3.2.

If we label $h:]-1,1[\longrightarrow \mathbb{R}$ as the height function, i.e.,

$$
h(r)=2 \operatorname{Re}\left(\int_{\delta_{1}} \tau_{r}\right)
$$

then a straightforward computation gives

$$
h(r)=\frac{\sqrt{2}}{n} \int_{\arccos (-r)}^{\pi} \frac{d t}{\sqrt{-r-\cos (t)}}>0,
$$

where we are using the branch of arccos which maps ] - 1, 1[ into ]0, $\pi[$.

In what follows, and up to homotheties, we suppose

$$
B=1 / h(r)
$$

In other words, and from Lemma 3.2, we are normalizing the immersion $X$ in such a way that the distance between the planes $\pi_{1}$ and $\pi_{2}$ is 1 (i.e., $k=1 / 2)$. 
On the other hand and taking into account Lemma 3.2 and the symmetries of $X(M)$, the oriented distance $d(r)$ between $\ell_{0}^{+}$and $\ell_{0}^{-}$is given by:

$$
d(r)=-2 \operatorname{Re}\left(\int_{\delta} \phi_{1}\right)
$$

This means that:

- $|d|$ is the distance between $\ell_{0}^{+}$and $\ell_{0}^{-}$.

- $d>0$ if and only if $\ell_{i}^{+}$and $\ell_{i}^{-}$do not intersect, $i=1,2$. See Figure 2.

- $d=0$ if and only if $\ell_{0}^{+}=\ell_{0}^{-}$(i.e., the end points of $\ell_{i}^{+}$and $\ell_{i}^{-}$coincide, $i=1,2)$.

- $d<0$ if and only if the half-lines $\ell_{i}^{+}$and $\ell_{i}^{-}$intersect at an interior point, $i=1,2$.

See Figure 10.

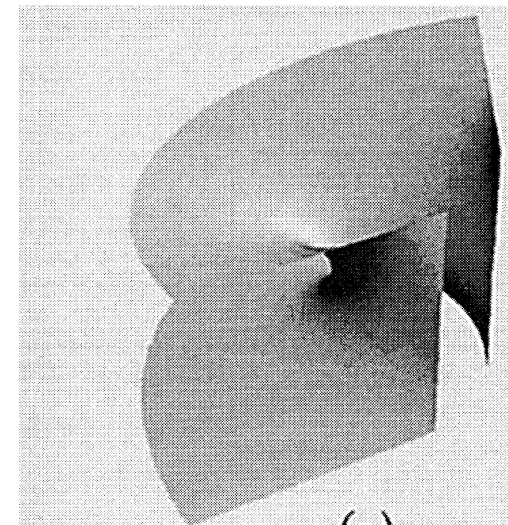

(a)

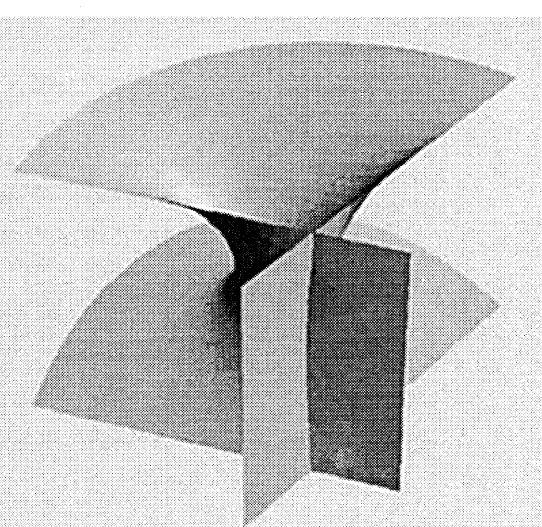

(b)

Figure 10: (a) A surface $X(M)$ for $\theta=2 \pi / 3$ and $d>0$. (b) A surface $X(M)$ for $\theta=2 \pi / 3$ and $d<0$. 


\subsection{The properness and convex hull property.}

Next lemma is devoted to study some topological and algebraic properties of $X$.

Lemma 2. The minimal immersion $X: M \rightarrow \mathbb{R}^{3}$ verifies:

1. $X$ is proper,

2. If $\theta \neq \pi, X(M)$ is contained in the convex hull, $\mathcal{E}(\Gamma)$, of $\Gamma$.

3. If $\theta=\pi, X(M)$ is contained in the intersection of the slab $-1 / 2 \leq$ $x_{3} \leq 1 / 2$ and one of the two halfspaces determined by the plane containing $\ell_{i}^{+}, \ell_{i}^{-}, i=0,1,2$.

Proof. Firstly, we are going to study the behaviour of $X$ around $E_{1}=(0,1)=$ $z^{-1}(0)$.

Taking into account the choice of the branch of $\varphi$, one has that

$$
\phi_{3}=-B z^{\frac{n}{2}-1} d z+A(z) d z
$$

where the branch of $z^{n / 2}$ satisfies $1^{n / 2}=1$. So

$$
\begin{aligned}
-\frac{i}{z} \phi_{3} & =i B z^{\frac{n}{2}-2} d z-i \frac{A(z)}{z} d z, \\
i z \phi_{3} & =-i B z^{\frac{n}{2}} d z+i z A(z) d z,
\end{aligned}
$$

where $A(z)$ is holomorphic around $E_{1}=z^{-1}(0)$ in $M$, and satisfies

$$
\lim _{z \rightarrow 0} \frac{A(z)}{z^{\frac{3 n}{2}-1}} \neq 0, \infty
$$

We define

$$
\begin{aligned}
& F(z)=-\frac{i}{2} \int \frac{1}{z} \phi_{3}=i \frac{B}{n-2} z^{\frac{n}{2}-1}+F_{1}(z), \\
& G(z)=\frac{i}{2} \int z \phi_{3}=-i \frac{B}{n+2} z^{\frac{n}{2}+1}+G_{1}(z), \\
& H(z)=\int \phi_{3}=-\frac{B}{n} z^{\frac{n}{2}}+H_{1}(z),
\end{aligned}
$$

where the limits

$$
\lim _{z \rightarrow 0} \frac{F_{1}(z)}{z^{\frac{3 n}{2}-1}}, \lim _{z \rightarrow 0} \frac{G_{1}(z)}{z^{\frac{3 n}{2}+1}}, \lim _{z \rightarrow 0} \frac{H_{1}(z)}{z^{\frac{3 n}{2}}}
$$


exist and are non zero. Then, $X$ can be expressed locally around $E_{1}$ as

$$
\begin{aligned}
X(z) & =\left(X_{1}(z), X_{2}(z), X_{3}(z)\right)=(\overline{F(z)}-G(z), \operatorname{Re}(H(z))) \\
& =\left(-\frac{i B}{n-2} z^{\frac{n}{2}-1}, 0\right)+\left(O_{1}(z), O_{2}(z), O_{3}(z)\right),
\end{aligned}
$$

where $O_{i}(z) /|z|$ is a bounded function in a neighbourhood of $E_{1}, i=1,2,3$. Equation (12) implies that for any sequence $\left\{P_{m}\right\}$ in $M$ converging $E_{1}$, the sequence $\left\{X\left(P_{m}\right)\right\}$ diverges in $\mathbb{R}^{3}$. Taking this and the symmetry $S_{h}$ into account, the same occurs for sequences converging to $E_{2}=(\infty, \infty)=$ $z^{-1}(\infty)$. Hence, we obtain that $X$ is proper.

On the other hand, (12) yields that the $\operatorname{limits} \lim _{P \rightarrow E_{1}} X_{3}(P)$ and $\lim _{P \rightarrow E_{2}} X_{3}(P)$ exist. Using Lemma 3.2 we deduce

$$
\lim _{P \rightarrow E_{1}} X_{3}(P)=\frac{1}{2}, \quad \lim _{P \rightarrow E_{2}} X_{3}(P)=-\frac{1}{2} .
$$

As $\Gamma$ lies in the slab $-1 / 2 \leq x_{3} \leq 1 / 2$, then we can use (13) and Theorem 2 to obtain that $X(M)$ is contained in this slab.

Label $\widehat{\sigma}^{+}$(resp. $\widehat{\sigma}^{-}$) as the plane containing $\ell_{1}^{+}$and $\ell_{2}^{+}$(resp. $\ell_{1}^{-}$and $\left.\ell_{2}^{-}\right)$.

If $d<0$, we denote $\sigma^{+}$(resp. $\sigma^{-}$) as the plane parallel to $\widehat{\sigma}^{+}$(resp. $\widehat{\sigma}^{-}$) containing $\ell_{0}^{-}$(resp. $\left.\ell_{0}^{+}\right)$.

In case $d \geq 0$, we put $\sigma^{+}=\widehat{\sigma}^{+}$and $\sigma^{-}=\widehat{\sigma}^{-}$.

Label also $\sigma_{0}$ to the plane parallel to $x_{2}=0$ containing $\ell_{0}^{+}$and $\ell_{0}^{-}$.

Let $\mathcal{H}^{+}$and $\mathcal{H}^{-}$be the open half spaces determined by $\sigma^{+}$and $\sigma^{-}$, respectively, and containing $\Gamma$. Let $\mathcal{H}_{0}$ denote the closed half space determined by $\sigma_{0}$ and containing $\Gamma$ too. Label $\mathcal{S}$ as the slab $x_{3}^{-1}([-1 / 2,1 / 2])$, and finally denote $\mathcal{E}=\mathcal{H}^{+} \cap \mathcal{H}^{-} \cap \mathcal{H}_{0} \cap \mathcal{S}$. Then, a straightforward argument gives that

- If $\theta \neq \pi$ and $d<0$, then $\mathcal{E}(\Gamma)=\mathcal{E} \cup \ell_{0}^{+} \cup \ell_{0}^{-}$.

- If $\theta \neq \pi$ and $d \geq 0$, then $\mathcal{E}=\overline{\mathcal{E}}$.

On the other hand, consider $\left\{P_{m}\right\}$ a sequence in $M$ converging to $E_{1}$, and assume that $\left\{\arg \left(\left(X_{1}\left(P_{m}\right), X_{2}\left(P_{m}\right)\right)\right\}\right.$ converges to $\theta_{0} \in[0,2 \pi[$. Let us prove that $\theta_{0} \in[\pi / 2-\theta / 2, \pi / 2+\theta / 2]$.

Indeed, from (12) and for $m$ large enough, one has that

$$
\left(X_{1}\left(P_{m}\right), X_{2}\left(P_{m}\right)\right)=\frac{i B}{2-n} z^{\frac{n}{2}-1}\left(P_{m}\right)+q_{m},
$$


where $\left\{q_{m}\right\}$ is bounded. As $\arg \left(z\left(P_{m}\right)\right) \in[-\pi / n, \pi / n]$, then

$$
\begin{aligned}
& \arg \left(\frac{i B}{2-n} z^{\frac{n}{2}-1}\left(P_{m}\right)\right) \in\left[\frac{\pi}{2}-\frac{(2-n) \pi}{2 n}, \frac{\pi}{2}+\frac{(2-n) \pi}{2 n}\right] \\
& =[\pi / 2-\theta / 2, \pi / 2+\theta / 2] .
\end{aligned}
$$

Since $q_{m}$ is bounded and $\left(X_{1}\left(P_{m}\right), X_{2}\left(P_{m}\right)\right)$ is divergent, we deduce that $\theta_{0} \in[\pi / 2-\theta / 2, \pi / 2+\theta / 2]$. This combined with equation (13) gives

$$
\lim _{m \rightarrow \infty} \operatorname{dist}\left(P_{m}, \mathcal{E}(\Gamma)\right)=0,
$$

and this equation holds for arbitrary sequences converging to $E_{1}$. By using the symmetry $S_{h}$, the equation (14) holds for sequences converging to $E_{2}$ too.

Let $\kappa$ be a plane which is not parallel to $\sigma^{+}$and $\sigma^{-}$, and not intersecting $\Gamma$. Let $\mathcal{K}$ denote the closed half space determined by $\kappa$ not containing $\Gamma$. Taking into account that $X$ is proper and (14), we have that $\mathcal{K} \cap X(M)$ is compact, and so, by an elementary consequence of Theorem 2 , it is empty. This proves Assertion 2 in the Lemma.

By a continuity argument, it is not hard to deduce Assertion 3 and complete the proof.

Remark 4. By using the same Weierstrass data, (4), for $n \in[2 / 3,1[$ and suitable $r \in]-1,1$, we can construct minimal examples with the same boundaries, which are not contained in their convex-hull. See Figure 7.

\subsection{Embeddedness.}

The following Lemmas are devoted to study under what conditions the immersion $X$ is an embedding.

Let $M^{+}=\{(z, w) \in M / \operatorname{Im}(z) \geq 0\}$ and $M^{-}=\{(z, w) \in M / \operatorname{Im}(z) \leq$ $0\}$, and define $\rho$ as the lift to $M$ of the divergent curve $] 0,+\infty[$ in $z(M)$. We parametrize $\rho$ as follows:

$$
\left.\rho(t)=z^{-1}(t), \quad t \in\right] 0,+\infty[.
$$

Obviously, the surfaces $M^{+}$and $M^{-}$are topologically a closed disk minus two boundary points. Furthermore,

$$
M=M^{+} \cup M^{-}, M^{+} \cap M^{-}=\rho
$$

and

$$
\partial\left(M^{+}\right)=\rho \cup \gamma_{1}^{+} \cup \gamma_{2}^{+} \cup \gamma_{0}^{+}, \partial\left(M^{-}\right)=\rho \cup \gamma_{1}^{-} \cup \gamma_{2}^{-} \cup \gamma_{0}^{-}
$$




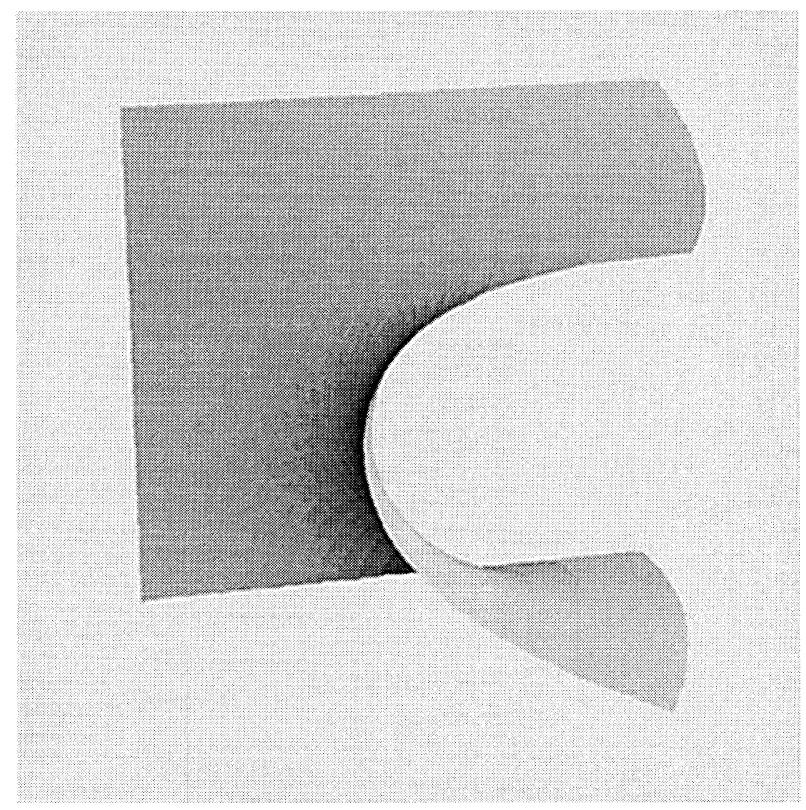

Figure 11: The surface $X\left(M^{-}\right)$when $\theta=\pi / 3$ and $r=0.8$.

Lemma 3. If $\theta \in] 0, \pi\left[\right.$, the surfaces $X\left(M^{+}\right)$and $X\left(M^{-}\right)$are graphs on the plane $x_{1}=0$. If $\theta=\pi$, the surfaces $X\left(M^{+}\right)-\left(\ell_{1}^{+} \cup \ell_{2}^{+}\right)$and $X\left(M^{-}\right)-\left(\ell_{1}^{-} \cup\right.$ $\left.\ell_{2}^{-}\right)$are graphs on the plane $x_{1}=0$.

Proof. Suppose first that $\theta \neq \pi$. Note that $\left.\phi_{3} / d t<0, t \in\right] 0,+\infty[$, and so $\left.X_{3}\right|_{\rho}$ is injective. Moreover, from (10) and (13),

$$
\lim _{t \rightarrow 0} X_{3}(\rho(t))=1 / 2, \quad \lim _{t \rightarrow+\infty} X_{3}(\rho(t))=-1 / 2 .
$$

On the other hand, $\rho$ is the fixed point set of $S_{v}$. Hence, $X(\rho)$ lies in the plane $x_{1}=0$.

By using the symmetry $S_{v}$, it suffices to prove that $X\left(M^{+}\right)$is a graph on $x_{1}=0$.

Let $\mathfrak{p}_{1}$ denote the orthogonal projection on the plane $x_{1}=0$. Since $X(M) \subset \mathcal{E}(\Gamma)$ and $X(M) \cap \mathcal{E}(\Gamma)=\Gamma$ (see Theorem 2), then

$$
\bigcup_{i=0}^{2} \mathfrak{p}_{1}\left(\ell_{i}^{+}\right) \subset \partial\left(\mathfrak{p}_{1}\left(M^{+}\right)\right),
$$


and $\mathfrak{p}_{1}\left(M^{+}\right)$is contained in the closed domain $W=\mathfrak{p}_{1}(\mathcal{E}(\Gamma))$ of the plane $x_{1}=0$, bounded by $\bigcup_{i=0}^{2} \mathfrak{p}_{1}\left(\ell_{i}^{+}\right)$. Let $W_{0}$ denote the closed domain in $W$ bounded by $X(\rho) \cup\left(\bigcup_{i=0}^{2} \mathfrak{p}_{1}\left(\ell_{i}^{+}\right)\right)$, and label $W_{1}=W-W_{0}$. It is clear that the open domain $W_{1}$ is bounded by the curve $X(\rho)$.

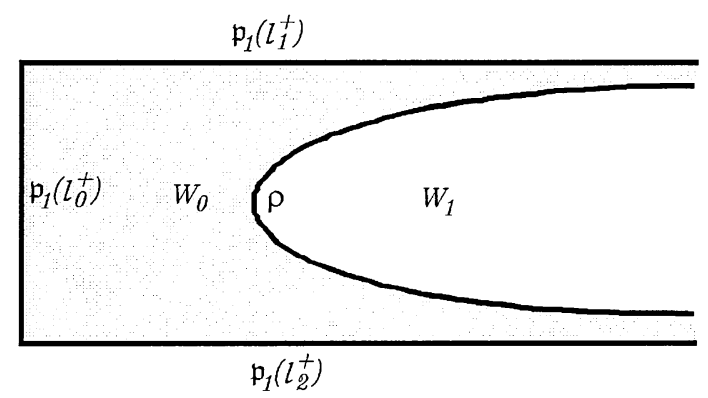

Figure 12: The domain $\mathfrak{p}_{1}\left(M^{+}\right)$.

As the normal vector $N(P)$ at any point $P \in M^{+}-\rho$ does not lie in the plane $x_{1}=0$, then it is not hard to see that

$$
\left.\mathfrak{p}_{1}\right|_{X\left(M^{+}-\rho\right)}: M^{+}-\rho \longrightarrow \mathfrak{p}_{1}\left(M^{+}\right)
$$

is a local diffeomorphism.

In particular, $\mathfrak{p}_{1}\left(M^{+}\right) \cap W_{1}$ is an open subset of $W_{1}$. However, as $X$ is proper (see Lemma 3.3), we have that $\mathfrak{p}_{1}\left(M^{+}\right) \cap W_{1}$ is also a closed subset of $W_{1}$. Since $W_{1}$ is connected, either $\mathfrak{p}_{1}\left(M^{+}\right) \cap W_{1}=W_{1}$ or $\mathfrak{p}_{1}\left(M^{+}\right) \cap W_{1}=\emptyset$. On the other hand, recall that $X_{3}$ extends continuously to the ends $\left\{E_{1}, E_{2}\right\}$ and $X_{3}\left(E_{1}\right)=1 / 2, X_{3}\left(E_{2}\right)=-1 / 2$. This easily implies that only a compact subset of the $x_{2}$-axis is contained in $\mathfrak{p}_{1}\left(M^{+}\right)$, and so $\mathfrak{p}_{1}\left(M^{+}\right) \cap W_{1}=\emptyset$.

A similar argument yields $\mathfrak{p}_{1}\left(M^{+}\right) \cap W_{0}=W_{0}$, i.e., $\mathfrak{p}_{1}\left(M^{+}\right)=W_{0}$. Now it is easy to deduce that

$$
\left.\mathfrak{p}_{1}\right|_{X\left(M^{+}\right)}: M^{+} \longrightarrow \mathfrak{p}_{1}\left(M^{+}\right)
$$

is a submersion. 
Since $X$ is proper and $X(M) \subset \mathcal{E}(\Gamma)$, the map $\left.\mathfrak{p}_{1}\right|_{X\left(M^{+}\right)}: M^{+} \rightarrow \mathfrak{p}_{1}\left(M^{+}\right)$ is also proper, and so, it is a covering. Since $\mathfrak{p}_{1}\left(M^{+}\right)=W_{0}$ is simplyconnected, $\left.\mathfrak{p}_{1}\right|_{X\left(M^{+}\right)}: M^{+} \rightarrow \mathfrak{p}_{1}\left(M^{+}\right)$is a homeomorphism. This proves the Lemma for $\theta<\pi$.

If $\theta=\pi$, use a continuity argument. This concludes the proof.

Lemma 4. If $d(r) \geq 0$, then $X\left(M^{+}\right)$(resp. $\left.X\left(M^{-}\right)\right)$is contained in the half space $x_{1} \geq 0$ (resp. $\left.x_{1} \leq 0\right)$.

In particular, $d(r)>0$ implies that $X$ is an embedding. If $d(r)=0$, then $\left.X\right|_{M-\gamma^{+}}$and $\left.X\right|_{M-\gamma^{-}}$are injective.

Proof. In this proof we use similar ideas to those in Lemma 3.3.

By using the symmetry $S_{v}$, it suffices to prove the Lemma for $X\left(M^{+}\right)$.

First, note that $d(r) \geq 0$ and Lemma 3.2 imply that $X\left(\partial\left(M^{+}\right)\right)$lies in the half space $x_{1} \geq 0$.

Let $\left\{Q_{m}\right\}$ be a sequence in $M^{+}$converging to $E_{1}$, such that $\left\{\arg \left(\left(X_{1}\left(Q_{m}\right), X_{2}\left(Q_{m}\right)\right)\right\}\right.$ converges to $\theta_{0} \in\left[0,2 \pi\left[\right.\right.$. Let us see that $\theta_{0} \in$ $[0, \pi / 2+\theta / 2]$.

Indeed, if $m$ is large enough, then from (12) one has that

$$
\left(X_{1}\left(Q_{m}\right), X_{2}\left(Q_{m}\right)\right)=\frac{i B}{2-n} z^{\frac{n}{2}-1}\left(Q_{m}\right)+p_{m}
$$

where $\left\{p_{m}\right\}$ is bounded. As $\arg \left(z\left(Q_{m}\right)\right) \in[-\pi / n, 0]$, then

$$
\arg \left(\frac{i B}{2-n} z^{\frac{n}{2}-1}\left(Q_{m}\right)\right) \in\left[0, \frac{\pi}{2}+\frac{(2-n) \pi}{2 n}\right]=[0, \pi / 2+\theta / 2] .
$$

As $p_{m}$ is bounded and $\left(X_{1}\left(Q_{m}\right), X_{2}\left(Q_{m}\right)\right)$ diverges, we have $\theta_{0} \in$ $[0, \pi / 2+\theta / 2]$. As a consequence, we obtain also that

$$
\lim _{m \rightarrow \infty} \operatorname{dist}\left(Q_{m},\left\{\left(x_{1}, x_{2}, x_{3}\right) \in \mathbb{R}^{3} / x_{1} \geq 0\right\}\right)=0 .
$$

By using $S_{h}$, then a symmetric equation to (15) holds for sequences converging to $E_{2}$.

Bcause of equation (15) and the properness of $X$, the same argument as at the end of the proof of Lemma 3.3 shows that $X\left(M^{+}\right)$lies in the convex hull of its boundary. This concludes the proof. 


\subsection{The analytical properties of the opening function $d(r)$.}

In order to understand the global behaviour of our family of surfaces, we are going to carry out a careful analysis of the function $d:]-1,1[\rightarrow \mathbb{R}$.

Define $\widehat{\delta}$ as the lift of the oriented curve $e^{t i / n}, t \in\left[-x_{0}, x_{0}\right]$, in the $z$-plane, and note that

$$
d(r)=-\operatorname{Re}\left(\int_{\widehat{\delta}} \phi_{1}\right) .
$$

It is clear that $\left(S_{h}\right)_{*}(\widehat{\delta})=\widehat{\delta}$ and $\left(S_{v}\right)_{*}(\widehat{\delta})=-\widehat{\delta}$.

Therefore, from 6 , we deduce that

$$
\int_{\widehat{\delta}} \phi_{1}=\overline{\int_{\widehat{\delta}} \phi_{1}}=-i \int_{\widehat{\delta}} z \phi_{3} .
$$

If we define $f:]-1,1[\longrightarrow \mathbb{R}$

$$
f(r)=i \int_{\widehat{\delta}} z \tau_{r}=2 \operatorname{Real}\left(i \int_{\delta} z \tau_{r}\right)=\frac{\sqrt{2}}{n} \int_{0}^{\arccos (-r)} \frac{\cos (t / n)}{\mid \sqrt{\cos (t)+r \mid}} d t
$$

then $d(r)$ is given by

$$
d(r)=\frac{f(r)}{h(r)}
$$

To obtain the last equality in (16), we have taken into account the choice of the branch of $\varphi$.

Lemma 5. The functions $f, h$, and $d$ satisfy the following differential equations:

$$
\begin{aligned}
& \text { 1. }\left(1-r^{2}\right) f^{\prime \prime}(r)-2 r f^{\prime}(r)+\frac{4-n^{2}}{4 n^{2}} f(r)=0 . \\
& \text { 2. }\left(1-r^{2}\right) h^{\prime \prime}(r)-2 r h^{\prime}(r)-\frac{1}{4} h(r)=0 . \\
& \text { 3. }-d(r)^{2}+n^{2}\left(2-3 n^{2}-2 r^{2}-n^{2} r^{2}\right) d^{\prime}(r)^{2} \\
& \quad+\left(4 d(r)-3 n^{2} d^{\prime \prime}(r)\right) n^{2}\left(-1+r^{2}\right) d^{\prime \prime}(r) \\
& \quad+2 n^{2} d^{\prime}(r)\left(2 r d(r)+n^{2} d^{(3)}(r)-2 n^{2} r^{2} d^{(3)}(r)+n^{2} r^{4} d^{(3)}(r)\right)=0 .
\end{aligned}
$$


Proof. Differentiating, one has

$$
\begin{gathered}
\frac{d^{2}}{d r^{2}}\left(\tau_{r}\right)-\frac{2 r}{1-r^{2}} \frac{d}{d r}\left(\tau_{r}\right)-\frac{1}{4\left(1-r^{2}\right)} \tau_{r}=d(\widehat{h}), \\
\frac{d^{2}}{d r^{2}}\left(z \tau_{r}\right)-\frac{2 r}{1-r^{2}} \frac{d}{d r}\left(z \tau_{r}\right)+\frac{4-n^{2}}{4 n^{2}\left(1-r^{2}\right)} z \tau_{r}=d(\widehat{f}),
\end{gathered}
$$

where $\widehat{h}$ and $\widehat{f}$ are the following meromorphic functions:

$$
\begin{aligned}
& \widehat{h}=\frac{z^{2 n}-1}{2 n\left(1-r^{2}\right) z^{n}\left(z^{n}+z^{-n}+2 r\right)^{3 / 2}}, \\
& \widehat{f}=z^{1-n} \frac{(n-2)-4 r z^{n}-(n+2) z^{2 n}}{2 n^{2}\left(r^{2}-1\right)\left(z^{n}+z^{-n}+2 r\right)^{3 / 2}} .
\end{aligned}
$$

The function $y=z^{n}+z^{-n}+2 r$ is well defined on $z(M)$. Let $M_{\varphi}$ denote the two-sheeted covering Riemann surface (with boundary) of $z(M)$ where $\varphi=\sqrt{y}$ is well defined, and label $p_{\varphi}: M_{\varphi} \rightarrow z(M)$ as the conformal covering map. Note that, in a natural way, $M$ is biholomorphic to the closure in $M_{\varphi}$ of $p_{\varphi}^{-1}(z(M-\partial(M)))$, and up this biholomorphism we can consider $M \subset M_{\varphi}$.

Let $\alpha$ be any simple closed curve in $z(M)$ winding once around $e^{i x_{0} / n}$ and $e^{-i x_{0} / n}$, and let $\widetilde{\alpha}$ be a lift of $\alpha$ to $M_{\varphi}$.

If $\psi$ is a 1 -form on $M_{\varphi}$, then up to a suitable choice of the orientation of $\alpha$ one has

$$
\int_{\widetilde{\alpha}} \psi=2 \int_{\widehat{\delta}} \psi
$$

Applying the above equality to the one-forms in (19) and integrating by parts, we obtain 1 .

To obtain 2 , integrate by parts (18) along any curve in $M$ homologous to $-\delta_{2}+\delta_{1}$, with the same end points that $-\delta_{2}+\delta_{1}$ and not passing through $\left( \pm e^{i x_{0} / n}, 0\right)$.

Moreover, using 1, 2 and (17), it is not hard to obtain 3.

Lemma 6. The function $d:]-1,1[\rightarrow \mathbb{R}$ satisfies:

1. It vanishes at only one point $\left.r_{\theta} \in\right]-1,1[$. Furthermore $d(r)$ is positive in $]-1, r_{\theta}[$ and negative in $] r_{\theta}, 1[$.

2. $\lim _{r \rightarrow-1} d(r)=0$. In particular, $d$ is bounded in $]-1, r_{\theta}[$. 
3. It has only a critical point $\left.r_{\theta}^{\prime} \in\right]-1, r_{\theta}[$ which is a maximum. In particular, $\left.\sharp\left[d^{-1}(\{x\})\right]=2, \forall x \in\right] 0, d\left(r_{\theta}^{\prime}\right)[$.

Proof. From (17), it is obvious that $d\left(r_{\theta}\right)=0$ if and only if $f\left(r_{\theta}\right)=0$.

In order to study the function $f$, we consider $\tilde{f}:]-1,1[\rightarrow \mathbb{R}$ given by

$$
\widetilde{f}(r)=\int_{0}^{+\infty} \sigma(r, z) d z
$$

where,

$$
\sigma(r, z)=\frac{2 m z^{\frac{2 m-3}{2}}\left(z^{m}-r\right)}{\left(z^{2 m}-2 r z^{m}+1\right)^{3 / 2}}, \quad m=\frac{n}{2-n} .
$$

Our purpose is to prove that $f(r)=\lambda \widetilde{f}$ for a suitable $\lambda>0$.

To do this, observe that

$$
\frac{d^{2} \sigma}{d r^{2}}-\frac{2 r}{1-r^{2}} \frac{d \sigma}{d r}+\frac{4-n^{2}}{4 n^{2}\left(1-r^{2}\right)} \sigma=\frac{d}{d z}\left(\frac{z^{-\frac{1}{2}+m} P(r, z)}{m\left(r^{2}-1\right)\left(1-2 r z^{m}+z^{2 m}\right)^{\frac{5}{2}}}\right)
$$

where:

$P(r, z)=-r-4 m r+z^{m}+6 m z^{m}+2 r^{2} z^{m}+2 m r^{2} z^{m}-3 r z^{2 m}-4 m r z^{2 m}+z^{3 m}$.

Similar ideas to those used in the proof of Lemma 3.5 show that $\tilde{f}$ satisfies Equation 1 in Lemma 3.5. Furthermore, it is not hard to check that

$$
\begin{aligned}
& f(0)=\frac{2 \sqrt{\pi}}{n \Gamma\left(1+\frac{2-n}{4 n}\right) \Gamma\left(\frac{3 n-2}{4 n}\right)}, \quad f^{\prime}(0)=\frac{4 \sqrt{\pi}}{n \Gamma\left(\frac{2+n}{4 n}\right) \Gamma\left(\frac{n-2}{4 n}\right)} \\
& \tilde{f}(0)=\frac{2 \Gamma\left(\frac{4 m-1}{4 m}\right) \Gamma\left(\frac{2 m+1}{4 m}\right)}{\sqrt{\pi}}, \quad \tilde{f}^{\prime}(0)=-\frac{\Gamma\left(\frac{2 m-1}{4 m}\right) \Gamma\left(\frac{4 m+1}{4 m}\right)}{m \sqrt{\pi}},
\end{aligned}
$$

where $\Gamma$ is the classical Gamma function. Thus, it is straightforward to check that $\frac{f(0)}{f^{\prime}(0)}=\frac{\widetilde{f}(0)}{\tilde{f}^{\prime}(0)}$.

As $f(0)$ and $\tilde{f}(0)$ are positive, then there exists $\lambda>0$ such that $f(r)=$ $\lambda \tilde{f}(r)$, which implies that $f$ and $\widetilde{f}$ have got the same zeroes.

Then, it suffices to make a careful analysis of $\tilde{f}$.

By deriving, we obtain

$$
\frac{d \sigma}{d r}=-\frac{z^{m-3 / 2}}{\left(z^{2 m}-2 r z^{m}+1\right)^{3 / 2}}-2 \frac{d}{d z}\left(\frac{z^{m-1 / 2}}{\left(z^{2 m}-2 r z^{m}+1\right)^{3 / 2}}\right) .
$$


Hence, integrating by parts, we obtain

$$
\left.\tilde{f}^{\prime}(r)=-\int_{0}^{+\infty} \frac{z^{m-3 / 2}}{\left(z^{2 m}-2 r z^{m}+1\right)^{3 / 2}} d z<0, \forall r \in\right]-1,1[.
$$

This implies that $\tilde{f}$ has at most one zero.

From its definition, it is clear that $\widetilde{f}(r)>0, \forall r \in]-1,0]$.

In order to compute the limit of $\widetilde{f}$ at 1 , we use the Frobenius Method for the study of ordinary differential equations with singularities (see $[1, \S 4.8]$ ). Taking into account that $\tilde{f}$ is a solution of Equation 1 in Lemma 3.5 and the above mentioned method, we deduce that

$$
\widetilde{f}(r)=a \log (1-r) \varphi_{1}(r)+b \varphi_{2}(r)
$$

where $a, b \in \mathbb{R}, \varphi_{1}(1) \neq 0$ and $\varphi_{i}, i=1,2$, are analytic at $r=1$.

As $\lim _{r \rightarrow 1} \widetilde{f}^{\prime}(r)=-\infty$, we deduce $a \neq 0$, and so $\lim _{r \rightarrow 1} \widetilde{f}(r)=-\infty$. An intermediate value argument gives the existence of a unique zero $r_{\theta}$ of $\tilde{f}$.

As $f(0)>0$, then $d(r)>0, \forall r \in]-1, r_{\theta}[$, and so $d(r)<0, \forall r \in] r_{\theta}, 1[$. This concludes the proof of Statement 1 .

In order to prove 2 , observe that

$$
\lim _{r \rightarrow-1} \tilde{f}(r)=\int_{0}^{+\infty} \frac{2 m z^{\frac{2 m-3}{2}}\left(z^{m}+1\right)}{\left(z^{2 m}+2 z^{m}+1\right)^{3 / 2}} d z \in \mathbb{R}^{+},
$$

Moreover, it is clear that $0 \leq \sqrt{-r-\cos (t)} \leq \sqrt{1-\cos (t)}, t \in$ $[\arccos (-r), \pi]$, then

$$
h(r) \geq \frac{\sqrt{2}}{n} \int_{\arccos (-r)}^{\pi} \frac{d t}{\sqrt{1-\cos (t)}}=-\frac{2}{n} \log (\tan (\arccos (-r) / 4)),
$$

and so

$$
\lim _{r \rightarrow-1} h(r) \geq \lim _{r \rightarrow-1}\left(-\frac{2}{n} \log \left[\tan \left(\frac{\arccos (-r)}{4}\right)\right]\right)=+\infty .
$$

Both (23) and (24) give Assertion 2.

To obtain 3, note that 3 in Lemma 3.5 yields that $d^{\prime \prime}\left(r_{\theta}^{\prime}\right)<0$ for each critical point $\left.r_{\theta}^{\prime} \in\right]-1, r_{\theta}$ [ of $d$. Consequently, there exists only one critical point of $d$ in $]-1, r_{\theta}$ [ and it is a maximum. Obviously, $d\left(r_{\theta}^{\prime}\right)=\operatorname{Maximum}\{d(r): r \in]-1, r_{\theta}[\}$.

Hence, it is clear that $\left.\sharp\left[d^{-1}(\{x\})\right] \geq 2, \forall x \in\right] 0, d\left(r_{\theta}^{\prime}\right)\left[\right.$. If $\sharp\left[d^{-1}(\{x\})\right]>$ 2, for some $x \in] 0, d\left(r_{\theta}^{\prime}\right)$ [, then it implies the existence of a local minimum of $d$ in $]-1, r_{\theta}[$, which is absurd. This concludes the proof. 
Definition 1. For each $\theta \in] 0, \pi]$, we denote $d_{\theta}$ as the maximum of the opening function $\left.d(r), r \in]-1, r_{\theta}\right]$.

Remark 5. Since the function $d(n, r)$ is differentiable in $[1,2] \times]-1,1[$, then the function $\theta \longmapsto d_{\theta}$ is continuous in $\left.] 0, \pi\right]$.

In case $n=2$ (i.e., $\theta=0$ ), Theorem 2 implies that $0<d(r)<1$, $\forall r \in]-1,1[$, and Supremum $\{d(r), r \in]-1,1[\}=1$, (see Remark 2). Hence, the function $\theta \longmapsto d_{\theta}$ extends continuously to $[0, \pi]$, defining $d_{0}=1$.

For $n \in\left[1,2\left[\right.\right.$, it is straightforward to check from (17) that $\frac{\partial d}{\partial n}>0$, i.e.,

$$
\frac{\partial d}{\partial \theta}<0 .
$$

This implies that the function $\theta \longmapsto d_{\theta}$ is decreasing in $\left.] 0, \pi\right]$, and in particular,

$$
\left.0<d_{\pi}<d_{\theta}<1, \quad \theta \in\right] 0, \pi[\text {. }
$$

\subsection{Main Theorem.}

We summarize the information in the preceding subsections (Lemmas 3.2, $3.3,3.4,3.4$ and 3.5 ) in the the following:

Theorem 4. Let $\theta \in] 0, \pi]$ and $r \in]-1,1[$.

Consider the Weierstrass data given by (3) and (4), where $n=\frac{2 \pi}{\theta+\pi}$ and $B$ is given in (10). Define the immersion $X$ as in (5). Then $X$ satisfies:

(i) $X(M)$ is a properly immersed minimal disk with two boundary ends in $\mathbb{R}^{3}$.

(ii) $X(\partial(M))=\Gamma=\bigcup_{i=0}^{2}\left(\ell_{i}^{+} \cup \ell_{i}^{-}\right)$, where

1. The curves $\ell_{1}^{+}$and $\ell_{1}^{-}$are half-lines contained in a plane $x_{3}=$ $1 / 2$, they are symmetric with respect to the plane $x_{1}=0$, and the straight lines containing them meet at an angle $\theta$.

2. The curve $\ell_{2}^{+}$(resp. $\ell_{2}^{-}$) is the image under the symmetry with respect to the plane $x_{3}=0$ of $\ell_{1}^{+}\left(\right.$resp. $\left.\ell_{1}^{-}\right)$.

3. The curve $\ell_{0}^{+}$(resp. $\ell_{0}^{-}$) is the vertical segment joining the end points of $\ell_{1}^{+}$and $\ell_{2}^{+}$(resp. $\ell_{1}^{-}$and $\left.\ell_{2}^{-}\right)$.

(iii) If $\theta \in] 0, \pi[, X(M)$ is contained in the convex-hull $\mathcal{E}(\Gamma)$ of $\Gamma$. If $\theta=\pi$, $X(M)$ is contained in the intersection of the slab $-1 / 2 \leq x_{3} \leq 1 / 2$ and one of the two halfspaces determined by the plane containing $\ell_{i}^{+}$, $\ell_{i}^{-}, i=0,1,2$. 
(iv) $X(M)$ is invariant under the symmetries with respect to the planes $x_{1}=0$ and $x_{3}=0$.

(v) If $d$ is given as in (11), then this function of $r$ and $\theta$ measures the oriented distance between $\ell_{0}^{+}$and $\ell_{0}^{-}$. This means that

1. $|d|=\operatorname{dist}\left(\ell_{0}^{+}, \ell_{0}^{-}\right)$.

2. $d \leq 0$ if and only if $\ell_{i}^{+} \cap \ell_{i}^{-} \neq \emptyset, i=1,2$, and $d>0$ otherwise.

3. $d=0$ if and only if $\ell_{0}^{+}=\ell_{0}^{-}$.

(vi) For each $\theta \in] 0, \pi]$, there exists an only $\left.r_{\theta} \in\right] 0,1\left[\right.$ such that $d\left(r_{\theta}\right)=$ 0 . The function $d(r)$ is negative in $] r_{\theta}, 1[$ and positive in $]-1, r_{\theta}[$. Furthermore, $\lim _{r \rightarrow-1} d(r)=0$ and $d(r)$ has an unique critical point $\left.r_{\theta}^{\prime} \in\right]-1, r_{\theta}\left[\right.$. The number $d_{\theta}=d\left(r_{\theta}^{\prime}\right)$ is the maximum of the opening function $d(r)$ on $\left.]-1, r_{\theta}\right]$.

(vii) $X$ is an embedding if and only if $d>0$. If $d=0$ (i.e., $r=r_{\theta}$ ), then $\left.X\right|_{M-\gamma^{+}}$and $\left.X\right|_{M-\gamma^{-}}$are injective, where $\gamma^{+}$and $\gamma^{-}$are the connected components of $\partial(M)$.

The numbers $r \in]-1,1[$ and $\theta \in] 0, \pi]$ are analytical parameters of our family of surfaces. From now on we will refer to $X_{\theta r}$ as the immersion arising in above theorem for the values $r$ and $\theta$. Analogously we indicated by $M_{\theta} r$ the disc with the corresponding complex structure.

Then we can describe our family of surfaces $\mathcal{M}$ as follows:

$$
\left.\left.\mathcal{M}=\left\{X_{\theta r}: M_{\theta r} \rightarrow \mathbb{R}^{3} / r \in\right]-1,1[, \theta \in] 0, \pi\right]\right\} .
$$

Note that $\theta$ has a clear geometrical meaning: the angle that $\ell_{i}^{+}$makes with $\ell_{i}^{-}, i=1,2$. The meaning of parameter $r$ concerns to the underlying complex structure of the surface.

Remark 6. Elementary geometrical arguments give that the complete orientable minimal surface without boundary

$$
\widetilde{X}_{\theta r}: \widetilde{M}_{\theta r} \longrightarrow \mathbb{R}^{3}
$$

obtained from $X_{\theta r}\left(M_{\theta r}\right)$ by successive Schwarz reflections about straight lines is invariant under the vertical translation $T$ by vector $(0,0,2)$.

The case $\frac{\theta}{\pi} \in \mathbb{Q}$ and $r=r_{\theta}$ is specially interesting. The immersion $\widetilde{X}_{\theta}=\widetilde{X}_{\theta r_{\theta}}$ is singly periodic and the induced immersion

$$
Y_{\theta}: \widetilde{M}_{\theta} r_{\theta} /\langle T\rangle \longrightarrow \mathbb{R}^{3} /\langle T\rangle
$$


has four ends and finite total curvature. If we write $\frac{\pi}{\theta}=p / q, p, q \in \mathbb{N}$, $\operatorname{gcd}(p, q)=1$, then it is not hard to check that:

- If $p$ is even the surface $\widetilde{M}_{\theta} r_{\theta}$ is the two sheeted orientable covering of a nonorientable minimal surface properly immersed in $\mathbb{R}^{3}$. Moreover, $Y_{\theta}$ has four ends, its total curvature is $-8 \pi(p+q)$ and $\widetilde{M}_{\theta} r_{\theta} /\langle T\rangle$ has genus $2 p-1$. A fundamental piece bounded by straight lines of the surface $\widetilde{X}_{\theta}\left(\widetilde{M}_{\theta r_{\theta}}\right), \theta=\pi / 2$, is illustrated in Figure 13 .

Figure 13: A fundamental piece of the surface $\widetilde{X}_{\theta}\left(\widetilde{M}_{\theta r_{\theta}}\right)$, for $\theta=\pi / 2$, contained in the slab $-1 / 2 \leq x_{3} \leq 3 / 2$.

- If $p$ is odd the surface $X_{\theta r_{\theta}}\left(M_{\theta r_{\theta}}\right)$ is invariant under a translation $T^{\prime}$ by vector $(0,0,1)$, and the induced immersion

$$
Y_{\theta}^{\prime}: \widetilde{M}_{\theta r_{\theta}} /\left\langle T^{\prime}\right\rangle \longrightarrow \mathbb{R}^{3} /\left\langle T^{\prime}\right\rangle
$$

has two ends. Moreover, if $q$ is even (resp. $q$ is odd), $Y_{\theta}^{\prime}$ has total curvature $-8 \pi(p+q)$ (resp. $-4 \pi(p+q))$ and $\widetilde{M}_{\theta} r_{\theta} /\left\langle T^{\prime}\right\rangle$ has genus 
$2 p$ (resp. $p$ ). Figure 14 shows a fundamental piece of the surface $\widetilde{X}_{\theta}\left(\widetilde{M}_{\theta r_{\theta}}\right), \theta=\pi / 3$.

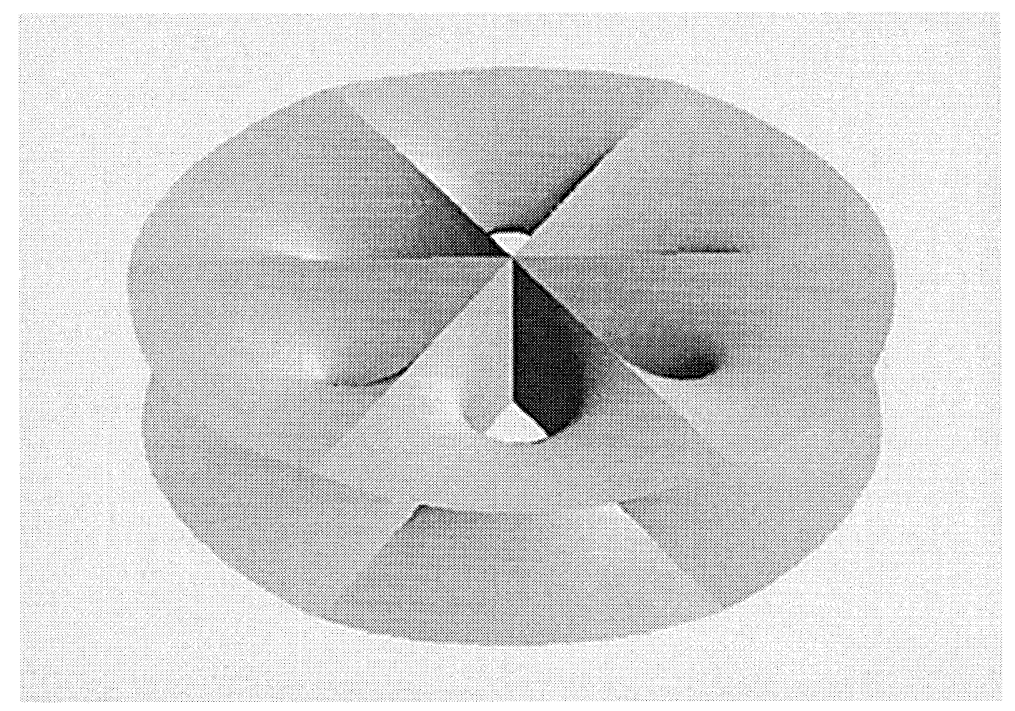

Figure 14: A fundamental piece of the surface $\widetilde{X}_{\theta}\left(\widetilde{M}_{\theta r_{\theta}}\right)$, for $\theta=\pi / 3$, contained in the slab $-1 / 2 \leq x_{3} \leq 1 / 2$.

- The ends of $Y_{\theta r_{\theta}}$ are embedded if and only if $\pi / \theta$ belongs to $\mathbb{N}$.

\subsection{Stability and limits.}

Finally, a few words about the stability of the surfaces $X_{\theta r}\left(M_{\theta r}\right), r \in$ ]$\left.-1, r_{\theta}\right]$.

From (16), it is straightforward to check that $f\left(\frac{n \pi}{2}\right)>0$. Since $\lim _{r \rightarrow 1} f(r)=-\infty$ (see the proof of Lemma 3.5), then $\left.r_{\theta} \in\right] \frac{n \pi}{2}, 1[$. Therefore, for $r=r_{\theta}$, the spherical image of the Gauss map of $X$ contains a hemisphere, and so this immersion is unstable.

However, and in the sense explained in the following proposition, the limit surface obtained as $r \rightarrow-1$ is stable.

Proposition 1. As set of points in $\mathbb{R}^{3}$, the surfaces $X_{\theta r}\left(M_{\theta r}\right)$ converge on compact subsets of $\mathbb{R}^{3}$, as $r \rightarrow-1$, to the union of the segment 
$[(0,0,-1 / 2),(0,0,1 / 2)]$ and the two parallel planar sectors in $\pi_{1}$ and $\pi_{2}$ determined by the planes $\cos \left(\frac{\theta}{2}\right) x_{1}-\sin \left(\frac{\theta}{2}\right) x_{2}=0$ and $\cos \left(\frac{\theta}{2}\right) x_{1}+\sin \left(\frac{\theta}{2}\right) x_{2}=0$ and contained in the half space $x_{2} \geq 0$.

Furthermore, for each $\epsilon \in] 0,1 / 2\left[\right.$, the surfaces $X_{\theta r}\left(M_{\theta r}\right) \cap\left\{\left|x_{3}\right| \leq\right.$ $1 / 2-\epsilon\}$ converge on compact subsets of $\mathbb{R}^{3}$, as $r \rightarrow-1$, to the segment $[(0,0,-1 / 2+\epsilon),(0,0,1 / 2-\epsilon)]$.

An example of these limit surfaces has been illustrated in Figure 6 .

Proof. For convenience, we write

$$
g_{r}=i z, \quad \eta_{r}=B(r) \frac{d z}{z^{2} \sqrt{z^{n}+z^{-n}+2 r}}
$$

and observe

$$
2 X_{\theta r}(P)=\operatorname{Re}\left(\int_{P_{0}}^{P}\left(\left(1-g_{r}^{2}\right) \eta_{r}, i\left(1+g_{r}^{2}\right) \eta_{r}, 2 g_{r} \eta_{r}\right)\right)
$$

for $r \in]-1,1\left[\right.$. Recall that $z\left(P_{0}\right)=1$.

Note that $\left.\left.z\left(M_{\theta r}\right)=z\left(M_{\theta r^{\prime}}\right), r, r^{\prime} \in\right]-1, r_{\theta}\right]$, and label $\Omega=z\left(M_{\theta r}\right)$, $\left.r \in]-1, r_{\theta}\right]$.

If we write $A=\mathbb{D} \cap \Omega$, then $\left.z\right|_{z^{-1}(A)}$ is injective, and so we identify the sets $z^{-1}(A)$ and $A$.

We deal with the convergence of the family of minimal surfaces $\left\{X_{\theta r}(\bar{A})\right\}$, as $r \rightarrow-1$.

Introduce the change of variable $z=B(r)^{\frac{2}{2-n}} x$. In the $x$-plane, $\bar{A}$ corresponds to the set $B(r)^{\frac{2}{n-2}} \cdot \bar{A}$, the Weierstrass data become

$$
g_{r}=i B(r)^{\frac{2}{2-n}} x, \quad \eta_{r}=-i \frac{d x}{x^{2-n / 2} \sqrt{B(r)^{\frac{4 n}{2-n}} x^{2 n}+2 r B(r)^{\frac{2 n}{2-n}} x^{n}+1}},
$$

and the initial condition is $P_{0} \equiv x\left(P_{0}\right)=B(r)^{\frac{2}{n-2}}$.

Taking into account that $\lim _{r \rightarrow-1} B(r)=0$ (see the proof of Lemma 3.5), then, as $r \rightarrow-1$, the set $B(r)^{\frac{2}{n-2}} \bar{A}$ converges to $\Omega$, and the Weierstrass data to

$$
g_{-1}=0, \quad \eta_{-1}=-i \frac{d x}{x^{2-n / 2}}
$$

Moreover, note that $\lim _{r \rightarrow-1} x\left(P_{0}\right)=\infty$.

Hence, if $r_{j}$ is a sequence converging to -1 as $j \rightarrow \infty$, and $P_{j} \in B\left(r_{j}\right)^{\frac{2}{n-2}}$. $\bar{A}$, then the sequence $X_{\theta r_{j}}\left(P_{j}\right)$ converges in $\mathbb{R}^{3}$ if and only if the sequence $P_{j}$ converges in $\Omega \cup\{\infty\}$. 
If $P_{j}$ converges to a finite point (in the $x$-plane), then $z\left(P_{j}\right)=$ $B\left(r_{j}\right)^{\frac{2}{2-n}} P_{j}$ converges to 0 in the $z$-plane. Thus, it is obvious that $X_{r_{j}}\left(P_{j}\right)$ converges to a point in $\pi_{1}$ (lying in the sector described in the statement of the lemma).

If $P_{j}$ converges to $\infty$ (in the $x$-plane), an analogous argument gives that $X_{\theta r_{j}}\left(P_{j}\right)$ converges to a point of the $x_{3}$-axis whose third coordinate lies in $[0,1 / 2]$. Furthermore, as $d(r) \rightarrow 0$ as $r \rightarrow-1$ (see Lemma 3.5), then, for any $q \in[(0,0,0),(0,0,1 / 2)]$, we can find a sequence $P_{j}$ as above converging to $q$.

By using the symmetry $S_{h}$, we conclude the proof of the first part of the proposition.

For the second part, take $\epsilon \in] 0,1 / 2[$. The third coordinate function of $X_{\theta r}$ is continuous at $(0,-1)$ and $(\infty,-1)$, as function of $(z, r)$. Then, we can find $\delta_{\epsilon}>0$ and a compact set $K_{\epsilon} \subset \Omega$, such that

$$
\left.z\left(X_{\theta r}^{-1}\left(\left\{\left|x_{3}\right| \leq 1 / 2-\epsilon\right\}\right)\right) \subset K_{\epsilon}, \quad \forall r \in\right]-1,-1+\delta_{\epsilon}[.
$$

Hence, if $r_{j}$ is a sequence converging to -1 as $j \rightarrow \infty$, and in the $x$-plane, $P_{j} \in B\left(r_{j}\right)^{\frac{2}{n-2}} \cdot\left(\bar{A} \cap K_{\epsilon}\right)$, then $P_{j}$ converges to $\infty$, and so, as we have mentioned above, the sequence $X_{\theta r_{j}}\left(P_{j}\right)$ converges to a point of $[(0,0,0),(0,0,1 / 2)]$.

Taking the symmetry $S_{h}$ into account once again, we conclude the proof.

\section{Properly immersed minimal surfaces in a wedge of a slab.}

By the strong halfspace theorem [3], a properly immersed minimal surface in a wedge of a slab has non empty boundary. In this section we prove that such a surface satisfies the convex hull property. Furthermore, we obtain some non-existence theorems for properly immersed minimal surfaces with planar boundaries.

First, we introduce some notation.

Let $L$ be the segment $\{(0,0, t): t \in]-1 / 2,1 / 2[\}$. Label

$$
W=\left\{\left(x_{1}, x_{2}, x_{3}\right) \in \mathbb{R}^{3}:-\frac{1}{2} \leq x_{3} \leq \frac{1}{2}\right\} .
$$

For $\theta \in] 0,2 \pi[$, we write

$$
W_{\theta}=\left\{\left(x_{1}, x_{2}, x_{3}\right) \in W-L: \arg \left(\left(x_{1}, x_{2}\right)\right) \in[0, \theta]\right\} \cup L .
$$


Define also

$$
\Sigma_{\theta}=\left\{\left(x_{1}, x_{2}, x_{3}\right) \in W-L: \arg \left(\left(x_{1}, x_{2}\right)\right)=\theta\right\} \cup L,
$$

where $\theta \in[0,2 \pi[$.

Recall that the surfaces $\left.X_{\theta r}\left(M_{\theta r}\right), r \in\right]-1, r_{\theta}$ [, constructed in Section 3 are contained in a wedge of angle $\theta \in] 0, \pi]$ of a horizontal slab. Furthermore the thickness of this slab is 1 . Thus, after a rigid motion (unique if $\theta \neq \pi$ ), we can assume that $\partial\left(X_{\theta r}\left(M_{\theta r}\right)\right) \subset \partial\left(W_{\theta}\right)$. In the case of $\theta=\pi$ we choose the rigid motion in such a way that $X_{\pi r}\left(M_{\pi r}\right)$ is symmetric with respect to the plane $x_{1}=0$.

For the sake of simplicity, we label $S_{\theta r}=X_{\theta r}\left(M_{\theta r}\right)$. Moreover, we write $S_{\theta}$ instead of $S_{\theta r_{\theta}}$.

We start with the following lemma:

Lemma 7. Let $M$ be a connected properly immersed minimal surface in the wedge $W_{\theta_{1}}, \theta_{1}<2 \pi$, and suppose that $\partial(M) \subset \Sigma_{0}$. Then $M$ is a planar region in $\Sigma_{0}$.

Proof. Up to a homothety and a translation in the direction of the $x_{1}$-axis, we can assume that:

- The distance from $\partial(M)$ to the planes $x_{3}=1 / 2$ and $x_{3}=-1 / 2$ is positive. As the immersion is proper, the distance from $M$ to the planes $x_{3}=1 / 2$ and $x_{3}=-1 / 2$ is also positive (see Theorem 2).

- There exists $\delta>0$ such that $x_{1} \geq \delta, \forall\left(x_{1}, x_{2}, x_{3}\right) \in \partial(M)$.

First suppose that $\theta_{1} \leq \pi$.

An application of Theorem 2 gives that $\Sigma_{\pi} \cap M=\emptyset$. Then, consider

$$
\theta_{0}=\operatorname{Infimum}\left\{\theta \in[0, \pi]: \Sigma_{\theta} \cap M=\emptyset\right\} .
$$

The theorem holds if and only if $\theta_{0}=0$.

We proceed by contradiction, and suppose $\theta_{0}>0$. Note that Theorem 2 gives $M \subset W_{\theta_{0}}$.

From Definition 1, it is clear that

$$
\left.\operatorname{dist}\left(L, \partial\left(S_{\theta_{0} r}\right)\right) \leq \frac{d_{\theta_{0}}}{2 \sin \left(\frac{\theta_{0}}{2}\right)}, \quad r \in\right]-1, r_{\theta_{0}}[.
$$




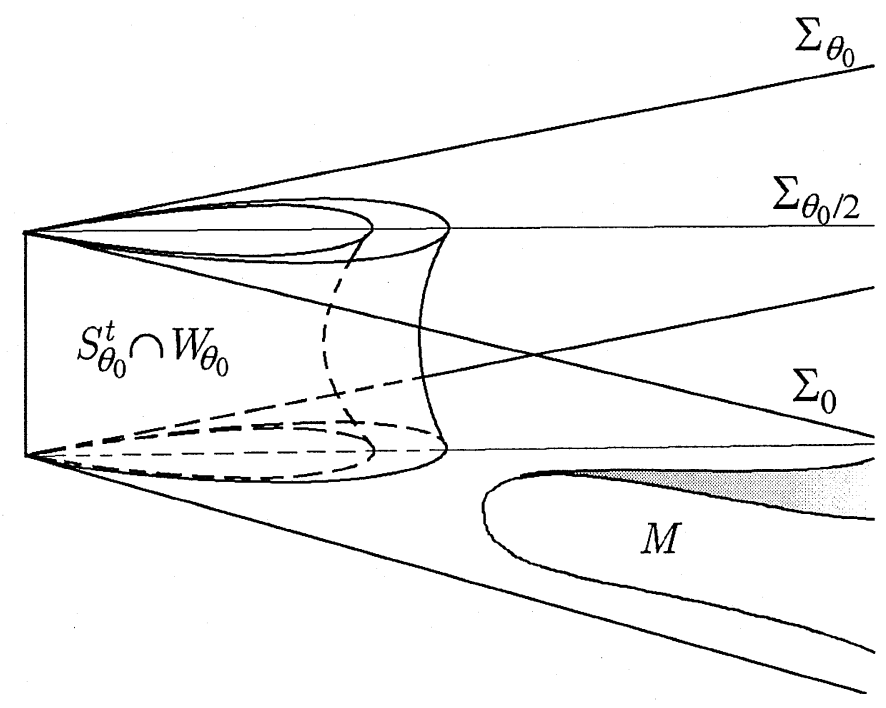

Figure 15: The surfaces $S_{\theta_{0}}^{t} \cap W_{\theta_{0}}$ and $M$.

Up to a translation in the direction of the $x_{1}$-axis, we can assume

$$
\delta>\frac{d_{\theta_{0}}}{2 \sin \left(\frac{\theta_{0}}{2}\right)},
$$

and so $\left.\left.\partial\left(S_{\theta_{0} r}\right) \cap M=\emptyset, \forall r \in\right]-1, r_{\theta_{0}}\right]$. In the following we prove that, in fact, $\left.\left.S_{\theta_{0} r} \cap M=\emptyset, \forall r \in\right]-1, r_{\theta_{0}}\right]$. Otherwise, we can find $\left.\left.r^{\prime} \in\right]-1, r_{\theta_{0}}\right]$ such that $S_{\theta_{0} r^{\prime}} \cap M \neq \emptyset$. On the other hand, taking into account that the surfaces are properly immersed, we use Proposition 3.7 to infer the existence of $\left.s \in]-1, r_{\theta}\right]$ such that $\left.\left.S_{\theta_{0} r} \cap M=\emptyset, \forall r \in\right]-1, s\right]$. Let $r^{\prime \prime}=\operatorname{Infimum}\{r \in$ ]$\left.\left.-1, r_{\theta_{0}}\right]: S_{\theta_{0} r} \cap M \neq \emptyset\right\}$. Since $S_{\theta_{0} r}$ and $M$ are properly immersed and do not have any contact at infinity, $\forall r \in]-1, r_{\theta_{0}}$ ], then $S_{\theta_{0} r^{\prime \prime}} \cap M \neq \emptyset$ and, obviously, $\left.S_{\theta_{0} r} \cap M=\emptyset, \forall r \in\right]-1, r^{\prime \prime}[$. Moreover, the above arguments imply that:

$$
\left(S_{\theta_{0} r^{\prime \prime}} \cap M\right) \cap\left(\partial\left(S_{\theta_{0} r^{\prime \prime}}\right) \cup \partial(M)\right)=\emptyset .
$$

Hence, Theorem 2 leads to a contradiction.

In particular, $S_{\theta_{0}} \cap M=\emptyset$.

Denote by $S_{\theta_{0}}^{t}$ the homothetic shrinking of $S_{\theta_{0}}$ by $t, t \geq 1$. It is clear that $S_{\theta_{0}}^{t}$ and $M$ do not contact at infinity. Taking into account that $S_{\theta_{0}}$ lies in $W_{\theta_{0}}$ and $x_{3}\left(\left(\partial\left(S_{\theta_{0}}\right)-L\right) \cap\left(\Sigma_{0} \cup \Sigma_{\pi}\right)\right)=\{-1 / 2,1 / 2\}$, it follows that $\partial\left(S_{\theta_{0}}^{t} \cap \overline{W_{\theta_{0}}}\right) \subset\left\{\left|x_{3}\right|=1 / 2\right\} \cup L$, and so $\left(S_{\theta_{0}}^{t} \cap M\right) \cap\left(\partial\left(S_{\theta_{0}}^{t}\right) \cup \partial(M)\right)=\emptyset$, $t \geq 1$. Reasoning as above, Theorem 2 implies that $S_{\theta_{0}}^{t} \cap M=\emptyset, t \geq 1$. 
For any $t>1$, the surface $S_{\theta_{0}}^{t} \cap W_{\theta_{0}}$ splits $W_{\theta_{0}}$ into two connected components, one of them bounded: $\operatorname{Int}\left(S_{\theta_{0}}^{t}\right)$, and the other one unbounded: $\operatorname{Ext}\left(S_{\theta_{0}}^{t}\right)$. For $t=1$, the surface $S_{\theta_{0}}$ also divides $W_{\theta_{0}}$ into two connected components, that we continue calling $\operatorname{Int}\left(S_{\theta_{0}}\right)$ and $\operatorname{Ext}\left(S_{\theta_{0}}\right)$.

Taking into account that $M$ is connected, $\partial(M) \subset \operatorname{Ext}\left(S_{\theta_{0}}^{t}\right)$ and $S_{\theta_{0}}^{t} \cap$ $M=\emptyset$, then it is easy to check that $M \subset \operatorname{Ext}\left(S_{\theta_{0}}^{t}\right), \forall t \in[1,+\infty[$. Therefore, $M \subset \Omega=\bigcap_{t \geq 1} \operatorname{Ext}\left(S_{\theta_{0}}^{t}\right)$. Taking into account that $\left(S_{\theta_{0}} \cap \Sigma_{\theta_{0} / 2}\right)-L$ is a connected arc diverging to both ends of $S_{\theta_{0}}$, it is not hard to deduce that $\Sigma_{\theta_{0} / 2} \cap \Omega=\emptyset$. Hence,

$$
M \cap \Sigma_{\theta_{0} / 2}=\emptyset .
$$

Since $M$ is connected, this fact contradicts the choice of $\theta_{0}$.

Finally, suppose that $\theta_{1}>\pi$. Let us see that in fact $M \subset W_{\pi}$. Otherwise, $M_{1}=M \cap \overline{W_{\theta_{1}}-W_{\pi}}$ is a surface contained in a wegde of angle $\theta_{1}-\pi<\pi$ whose boundary lies in $\Sigma_{\pi}$. So, by using the preceding reasoning, $M_{1}$ is a piece of a plane, which is absurd. This proves the lemma.

To state the next algebraic lemma, we will need the following notation.

For any $\vec{a} \in \mathbb{S}^{2}$ and $y \in \mathbb{R}^{3}$, define

$$
H_{\vec{a}}^{y}=\left\{x \in \mathbb{R}^{3}:\langle\vec{a},(x-y)\rangle \geq 0\right\} .
$$

As usual, we identify $\mathbb{R}^{2}=\left\{\left(x_{1}, x_{2}, x_{3}\right) \in \mathbb{R}^{3}: x_{3}=0\right\}$ and $\mathbb{S}^{1}=\mathbb{S}^{2} \cap \mathbb{R}^{2}$.

Lemma 8. Given $\vec{a} \in \mathbb{S}^{2}-\{(0,0, \pm 1)\}$ and $y \in \mathbb{R}^{3}$, there exists $\vec{a}_{0} \in \mathbb{S}^{1}$ and $z \in \mathbb{R}^{2}$ such that:

- $\left(H_{\vec{a}_{0}}^{z} \cap W\right) \subset\left(H_{\vec{a}}^{y} \cap W\right)$.

- The map $x \longmapsto\langle\vec{a},(x-y)\rangle$ is bounded in $\left(H_{\vec{a}}^{y}-H_{\vec{a}_{0}}^{z}\right) \cap W$.

The proof is merely an exercise. We will omit it.

We can now prove the main result of this section.

Theorem 5. Any connected properly immersed minimal surface in a slab wedge $\left.W_{\theta}, \theta \in\right] 0, \pi[$, lies in the convex hull of its boundary.

Proof. Let $M$ be a minimal surface satisfying the hypotheses of the theorem.

If $M$ is a piece of a plane the result obviously holds. Suppose that $M$ is not flat. 
Consider $\vec{a} \in \mathbb{S}^{2}$ and $y \in \mathbb{R}^{3}$ such that $\partial(M)$ lies in $H_{-\vec{a}}^{y}$. We have to prove that $M \subset H_{-\vec{a}}^{y}$ too.

We proceed by contradiction, and suppose that $M \cap\left(H_{\vec{a}}^{y}-\partial\left(H_{\vec{a}}^{y}\right)\right) \neq \emptyset$. Let $M^{\prime}$ be a connected component of $M \cap H_{\vec{a}}^{y}$. If $\vec{a}=(0,0, \pm 1)$, and taking into account that $\partial\left(M^{\prime}\right) \subset \partial\left(H_{\vec{a}}^{y}\right)$, it is easy to deduce from Theorem 2 that $M^{\prime}$ (and so $M$ ) is flat, which is absurd. In what follows, we assume that $\vec{a}$ is not vertical. Consider $\vec{a}_{0} \in \mathbb{S}^{1}$ and $z \in \mathbb{R}^{2}$ given by Lemma 4 when applied to $(\vec{a}, y)$. If $M^{\prime} \subset H_{\vec{a}}^{y}-H_{\vec{a}_{0}}^{z}$, then, from Theorem 2 we deduce, as above, that $M^{\prime}$ is a planar region, which is absurd. Hence, $M^{\prime} \cap H_{\vec{a}_{0}}^{z} \neq \emptyset$.

Let $M^{\prime \prime}$ be a connected component of $M^{\prime} \cap H_{\vec{a}_{0}}^{z}$, and take $\vec{b}_{0} \in \mathbb{S}^{1}$ orthogonal to $\vec{a}_{0}$. Since $\partial\left(M^{\prime \prime}\right) \subset\left(\partial\left(H_{\vec{a}_{0}}^{z}\right) \cap W_{\theta}\right)$ and $\theta<\pi$, then the set $\left\{\left\langle x, \vec{b}_{0}\right\rangle: x \in \partial\left(M^{\prime \prime}\right)\right\}$ is bounded either from above or below.

Up to a rigid motion, we can suppose that $\vec{a}_{0}=(0,1,0), z=(0,0,0)$ and $\partial\left(M^{\prime \prime}\right) \subset \Sigma_{0}$. Thus, we can apply Lemma 4 to deduce that $M^{\prime \prime}$ is a planar region. This is contrary to our assumptions, and concludes the proof.

Corollary 1. Let $M$ be a connected properly immersed minimal surface in the halfslab $W_{\pi}$. Assume $\left.\partial(M) \subset W_{\theta}, \theta \in\right] 0, \pi[$. Then $M$ lies in the convex hull of $\partial(M)$.

Proof. It suffices to prove that $M \subset W_{\theta}$. Indeed, if $M \not \subset W_{\theta}$, take a connected component $M^{\prime \prime}$ of $M-W_{\theta}$. Since $\partial\left(M^{\prime \prime}\right) \subset \Sigma_{\theta}$, then, up to a rigid motion, we can apply Lemma 4 to infer that $M^{\prime \prime}$ is a planar region in $\Sigma_{\theta}$, which is absurd.

Corollary 2. Let $M$ be a connected properly immersed minimal surface in the halfslab $\left.W_{\theta_{1}}, \theta_{1} \in\right] 0,2 \pi\left[\right.$. Assume $\left.\partial(M) \subset W_{\theta}, \theta \in\right] 0, \pi[$. Then $M$ lies in the convex hull of $\partial(M)$.

Proof. It suffices to prove that $M \subset W_{\pi}$, and use Corollary 4. Indeed, if $M \not \subset W_{\pi}$, take a connected component $M^{\prime \prime}$ of $M-W_{\pi}$. Since $\partial\left(M^{\prime \prime}\right) \subset \Sigma_{\pi}$ and $M^{\prime \prime}$ lies in a wedge of angle $\theta_{1}-\pi<\pi$, then, up to a rigid motion, we can apply Lemma 4 to infer that $M^{\prime \prime}$ is a planar region in $\Sigma_{\pi}$, which is absurd.

Theorem 4 can be extended to the case of $\theta=0$. For any $d \in] 0,+\infty[$, 
define

$$
C_{d}=\left\{\left(x_{1}, x_{2}, x_{3}\right) \in \mathbb{R}^{3}: 0 \leq x_{2} \leq d,-\frac{1}{2} \leq x_{3} \leq \frac{1}{2}\right\} .
$$

Theorem 6. Any connected properly immersed minimal surface in $C_{d}, d \in$ ] $0,+\infty[$, lies in the convex hull of its boundary.

The proof is as in Theorem 4.

Remark 7. Theorem 4 and Corollary 4 fail in the case of $\theta=\pi$. A simple counterexample is the surface $S_{\pi r}$, for any $\left.r \in\right]-1, r_{\pi}$ [. A suitable piece of a helicoid is another counterexample.

The following theorem represents another interesting application of the above techniques.

Theorem 7. Let $M$ be a connected properly immersed non flat minimal surface in a wedge $\left.\left.W_{\theta}, \theta \in\right] 0, \pi\right]$. Suppose that $\partial(M) \subset \Sigma_{0} \cup \Sigma_{\theta}$. Then,

$$
\operatorname{dist}(L, \partial(M)) \leq \frac{d_{\theta}}{2 \sin \left(\frac{\theta}{2}\right)},
$$

where $d_{\theta}$ is given in Definition 1.

Proof. First observe that the sets $\partial(M) \cap \Sigma_{0}$ and $\partial(M) \cap \Sigma_{\theta}$ are not empty. Otherwise, Lemma 4 says that $M$ is flat, which is absurd.

Suppose that $\operatorname{dist}(L, \partial(M))>\frac{d_{\theta}}{2 \sin \left(\frac{\theta}{2}\right)}$. After a suitable homothetic shrinking of $M$, we can suppose that $\operatorname{dist}(L, \partial(M))>\frac{d_{\theta}}{2 \sin \left(\frac{\theta}{2}\right)}$ and $\operatorname{dist}\left(\partial(M),\left\{\left|x_{3}\right|=1 / 2\right\}\right)>0$. ¿From Definition 1, it follows that $\operatorname{dist}(L,>$ $\left.\partial\left(S_{\theta r}\right)\right) \leq \frac{d_{\theta}}{2 \sin \left(>\frac{\theta}{2}\right)}$, and so we can use the surfaces $\left.S_{\theta r}, r \in\right]-1, r_{\theta}[$, as barriers for the maximum principle application. So, reasoning as in the proof of Lemma 4, we obtain $M \cap S_{\theta}=\emptyset$.

Consider the homothetic shrinking $S_{\theta}^{t}$ of $S_{\theta}$ by $t, t \geq 1$. Following the proof of Lemma 4, we use these surfaces as barriers to deduce that $M \cap \Sigma_{\theta / 2}=\emptyset$, which contradicts the fact that $M$ is connected.

The surface $S_{\theta r_{\theta}^{\prime}}$, corresponding to the maximum $d_{\theta}$ of the opening function, reaches the equality in Theorem 4 . Hence, this result is sharp.

Finally, we prove a non existence theorem for properly immersed non flat minimal surfaces with planar boundary. As stated in the introduction, 
this theorem is a generalization of a well-known result of Nitsche [12] in the non compact case (see also [14]).

For $d \in] 0,+\infty[$, label

$$
\begin{aligned}
& F_{d}^{0}=\left\{\left(x_{1}, x_{2}, x_{3}\right) \in C_{d}: x_{3}=-\frac{1}{2}\right\}, \\
& F_{d}^{1}=\left\{\left(x_{1}, x_{2}, x_{3}\right) \in C_{d}: x_{3}=\frac{1}{2}\right\} .
\end{aligned}
$$

Moreover, for any $d \in] 0,1\left[\right.$, and $t \in \mathbb{R}$, we denote $S_{d}^{t}$ as the Jenkins-Serrin graph over the rectangle $\{t\} \times[0, d] \times\left[-\frac{1}{2}, \frac{1}{2}\right]$ with boundary values:

- $-\infty$ on $\{t\} \times] 0, d\left[\times\left\{-\frac{1}{2}, \frac{1}{2}\right\}\right.$,

- 0 on $\{t\} \times\{0, d\} \times\left[-\frac{1}{2}, \frac{1}{2}\right]$.

See Remarks 2 and 5.

Theorem 8. Let $M$ be a connected properly immersed minimal surface in $\mathbb{R}^{3}$ satisfying:

1. $M \subset C_{d}$ and $\partial(M) \subset\left(F_{d}^{0} \cup F_{d}^{1}\right)$, where $0<d<1$,

2. $x_{1}>0, \forall\left(x_{1}, x_{2}, x_{3}\right) \in \partial(M)$.

Then $M$ is a planar region in $F_{d}^{0} \cup F_{d}^{1}$.

Proof.

Suppose $M$ is not flat.

From Theorem 4 we get $x_{1}>0, \forall\left(x_{1}, x_{2}, x_{3}\right) \in M$. Thus, $M \cap S_{d}^{t}=\emptyset$, $t \leq 0$. If it is necessary, we take $d^{\prime}, 1>d^{\prime}>d$, and translate $M$ in the direction of the $x_{2}$-axis, in such a way that the distance from $M$ to the planes $x_{2}=0$ and $x_{2}=d^{\prime}$ is positive. In particular, $M \cap S_{d^{\prime}}^{t}$ does not meet $\partial(M) \cup \partial\left(S_{d^{\prime}}^{t}\right), t>0$.

On the other hand, as the set $C_{d^{\prime}}-\left(\bigcup_{t \in \mathbb{R}} S_{d^{\prime}}^{t}\right)$ is included in $\left\{x_{3}=\right.$ $\left.-\frac{1}{2}\right\} \cup\left\{x_{3}=\frac{1}{2}\right\}$ and $M$ is not flat, then there is $t^{\prime}>0$ large enough such that $M \cap S_{d^{\prime}}^{t^{\prime}} \neq \emptyset$.

Label $t_{0}=\operatorname{Infimum}\left\{t \in \mathbb{R}: M \cap S_{d^{\prime}}^{t} \neq \emptyset\right\}$. From the above arguments, $t_{0} \in\left[0,+\infty\left[\right.\right.$. As $M$ and $S_{d^{\prime}}^{t}, t \in \mathbb{R}$, are properly immersed, then $S_{d^{\prime}}^{t_{0}} \cap M \neq \emptyset$. Therefore, $S_{d^{\prime}}^{t_{0}}$ and $M$ have an interior contact point, and so Theorem 2 leads to a contradiction. 


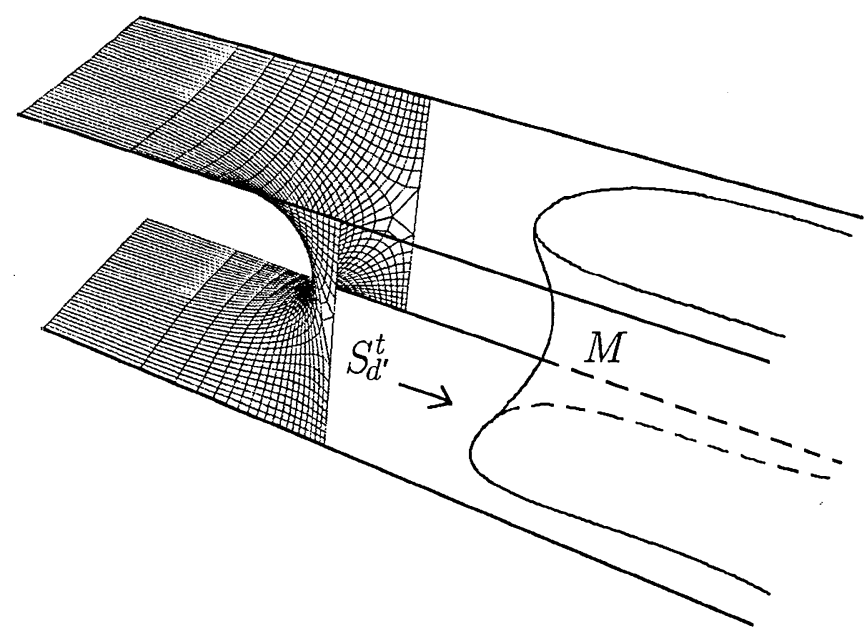

Figure 16: The surfaces $S_{d^{\prime}}^{t}, t \in \mathbb{R}$, and $M$.

\section{References.}

[1] E.A. Coddington and N. Levinson, Theory of Ordinary Differential Equations, Mc Graw-Hill Book Company, New York, 1955.

[2] G. Darboux, Tèorie gènèrale des surfaces, 1, Chelsea Publishing Co., New York, 1972.

[3] D. Hoffman and W.H. Meeks, III, The strong halfspace theorem for minimal surfaces, Invet. Math., 101 (1990), 373-377.

[4] H. Jenkins and J. Serrin, Variational problems of minimal surface type. II. Boundary value problems for the minimal surface equation, Arch. Rat. Mech. Anal., 21 (1966), 321-342.

[5] H. Karcher, Construction of minimal surfaces, Surveys in Geometry 1989/90, University of Tokyo, 1989. Also: Vorlesungsreihe Nr. 1, SFB 256, Bonn, 1989.

[6] F.J. Lopez, R. Lopez and R. Souam, Maximal surfaces of Riemann type in Lorentz-Minkowski space $\mathbb{L}^{3}$, Michigan Math. J., 47(3) (2000), 469-497. 
[7] F.J. Lopez and F. Martin, Uniqueness of properly embedded minimal surfaces bounded by straight lines, J. Austral. Math. Soc. Ser. A, 69(3) (2000), 362-402.

[8] F.J. Lopez, M. Ritore and F. Wei, A characterization of Riemann's minimal surfaces, J. Differential Geom., 47(2) (1997), 376-397.

[9] F.J. Lopez and F. Wei, Properly immersed minimal discs bounded by straight lines, Math. Ann., 318(4) (2000), 667-706.

[10] W.H. Meeks, III and H. Rosenberg, The global theory of doubly periodic minimal surfaces, Invent. Math., 97 (1989), 351-379.

[11] W. H. Meeks III and H. Rosenberg, The geometry and conformal structure of properly embedded minimal surfaces of finite topology in $\mathbb{R}^{3}$, Invent. Math., 114 (1993), 625-639.

[12] J.C.C. Nitsche, A supplement to the condition of J. Douglas, Rend. Circ. Matem. Palermo, Serie II, Tomo XIII, (1964).

[13] R. Osserman, A survey of minimal surfaces, Dover Publications, New York, second edition, 1986.

[14] W. Rossman, Minimal surfaces with planar boundary curves, Kyushu J. Math., 52 (1998), 209-225.

[15] R. Schoen, Uniqueness, symmetry and embeddedness of minimal surfaces, J. Differential Geom., 18 (1983), 791-809.

Departamento de Geometría y Topología

UNIVERSIDAD DE GRANADA

18071 Granada, Spain

E-mail address: fjlopez@goliat.ugr.es

Departamento de Geometría y Topología

UNIVERSIDAD DE GRANADA

18071 Granada, Spain

E-mail address: fmartin@goliat.ugr.es

Received December 1, 1998. 